\title{
CARATHÉODORY-JULIA TYPE THEOREMS FOR OPERATOR VALUED SCHUR FUNCTIONS
}

\author{
By \\ VLADIMIR BOLOTNIKOV AND ALEXANDER KHEIFETS
}

\begin{abstract}
We extend the Carathéodory-Julia theorem on angular derivatives as well as its higher order analogue established recently in [4] to the setting of contractive valued functions analytic on the unit disk. Carathéodory-Julia type conditions for an operator valued Schur-class function $w$ are shown to be equivalent to the requirement that every function from the de Branges-Rovnyak space associated with $w$ has certain directional boundary angular derivatives.
\end{abstract}

\section{Introduction}

The starting point of this paper is the classical Carathéodory-Julia theorem; in its formulation and in what follows, $\mathbb{D}$ and $\mathbb{T}$ denote the open unit disk and the unit circle, respectively, and $\mathcal{S}$ stands for the Schur class of analytic functions mapping $\mathbb{D}$ into $\overline{\mathbb{D}}$. We write $z \widehat{\rightarrow} t_{0}$ if a point $z \in \mathbb{D}$ approaches a boundary point $t_{0} \in \mathbb{T}$ nontangentially and $z \rightarrow t_{0}$ if $z$ approaches $t_{0}$ unrestrictedly in $\mathbb{D}$.

Theorem 1.1 (Carathéodory-Julia). Let $w \in \mathcal{S}$, let $t_{0} \in \mathbb{T}$ and let

$$
\liminf _{z \rightarrow t_{0}} \frac{1-|w(z)|^{2}}{1-|z|^{2}}<\infty .
$$

Then the nontangential limits

$$
d:=\lim _{z \breve{\rightarrow} t_{0}} \frac{1-|w(z)|^{2}}{1-|z|^{2}}<\infty, \quad w_{0}:=\lim _{z \breve{\rightarrow} t_{0}} w(z), \quad w_{1}:=\lim _{z \rightarrow t_{0}} w^{\prime}(z)
$$

exist and satisfy $\left|w_{0}\right|=1$ and $d=w_{1} t_{0} w_{0}^{*} \geq 0$.

The objective of this paper is to establish analogues of Theorem 1.1 for operatorvalued Schur functions. Let $\mathcal{L}(\mathcal{U}, \mathcal{Y})$ denote the algebra of bounded linear operators mapping a Hilbert space $\mathcal{U}$ into another Hilbert space $\mathcal{Y}$, and let $\mathcal{L}(\mathcal{Y}):=\mathcal{L}(\mathcal{Y}, \mathcal{Y})$. We denote by $\mathcal{S}(\mathcal{U}, \mathcal{Y})$ the Schur class of all $\mathcal{L}(\mathcal{U}, \mathcal{Y})$-valued functions analytic and 
contractive valued on $\mathbb{D}$. We write "w-lim", "s-lim" and "lim" for nontangential convergence in the weak, strong, and uniform operator topologies, respectively. By "the limit exists" we always mean that the limit is equal to a finite number (in the scalar case) or to a bounded operator (in the operator case). For $w \in \mathcal{S}(\mathcal{U}, \mathcal{Y})$, the quantities

$$
D^{w}(z)=\frac{\mathrm{I}_{\mathcal{Y}}-w(z) w(z)^{*}}{1-|z|^{2}} \quad \text { and } \quad \widetilde{D}^{w}(z)=\frac{\mathrm{I}_{\mathcal{U}}-w(z)^{*} w(z)}{1-|z|^{2}}
$$

are the operator valued analogues of the scalar valued term in (1.1). The boundedness of one of them does not (in general) imply the boundedness of another. Conditions (1.3) and (1.6) below are two possible extensions (in general not equivalent) of condition (1.1) to the operator valued setting. They lead to different operator analogues of Theorem 1.1.

Theorem 1.2. Let $w \in \mathcal{S}(\mathcal{U}, \mathcal{Y}), t_{0} \in \mathbb{T}$ and assume that

$$
\liminf _{z \rightarrow t_{0}}\left\langle D^{w}(z) y, y\right\rangle_{\mathcal{Y}}<\infty \quad \text { for every } y \in \mathcal{Y},
$$

where $D^{w}(z)$ is defined in (1.2). Then the strong limit $w_{0}^{*}:=\mathrm{s}_{z \rightarrow t_{0}} w(z)^{*}$ and the weak limits

$$
\begin{gathered}
D^{w}\left(t_{0}\right):=\underset{z}{\mathrm{w}-\lim } \underset{z \rightarrow t_{0}}{ } D^{w}(z), \\
q=\mathrm{w} \_\lim _{z \rightarrow t_{0}} w_{0} \widetilde{D}^{w}(z) w_{0}^{*} \quad \text { and } \quad f:=\mathrm{w}_{z \widehat{\rightarrow} t_{0}} w^{\prime}(z) w_{0}^{*} t_{0}
\end{gathered}
$$

exist and satisfy $w_{0} w_{0}^{*}=\mathrm{I}_{\mathcal{Y}}$ (i.e., $w_{0}$ is a coisometry) and $D^{w}\left(t_{0}\right)=f=q \geq 0$.

Theorem 1.3. Let $w \in \mathcal{S}(\mathcal{U}, \mathcal{Y}), t_{0} \in \mathbb{T}$ and assume that

$$
\liminf _{z \rightarrow t_{0}}\left\langle\widetilde{D}^{w}(z) u, u\right\rangle_{\mathcal{U}}<\infty \quad \text { for every } u \in \mathcal{U},
$$

where $\widetilde{D}^{w}(z)$ is defined in (1.2). Then the strong limit $w_{0}:=\mathrm{s}_{z \rightarrow t_{0}} w(z)$ and the weak limits $\widetilde{q}:=\mathrm{w}_{z \rightarrow \lim _{0}} w_{0}^{*} D^{w}(z) w_{0}, \widetilde{f}:=\mathrm{w}_{z \rightarrow t_{0}} w_{0}^{*} w^{\prime}(z) t_{0}$ and

$$
\widetilde{D}^{w}\left(t_{0}\right):=\underset{z}{\mathrm{w}-\lim } \widetilde{D} \widetilde{D}^{w}(z)
$$

exist and satisfy $w_{0}^{*} w_{0}=\mathrm{I}_{\mathcal{U}}$ (i.e., $w_{0}$ is an isometry) and $\widetilde{D}^{w}\left(t_{0}\right)=\widetilde{f}=\widetilde{q} \geq 0$.

In the matrix-valued setting, Theorems 1.2 and 1.3 appear in [9, Section 8] and [11], respectively. The proofs extend to the operator case in a fairly straightforward way; only establishing the existence of the strong limits $w_{0}^{*}$ (resp., $w_{0}$ ) requires some extra work. 
Remark 1.4. Theorem 1.3 can be obtained by applying Theorem 1.2 to the function $\widetilde{w}(z):=w(\bar{z})^{*} \in \mathcal{S}(\mathcal{Y}, \mathcal{U})$, since condition (1.6) for $w$ can be understood as a condition of (1.3) type for $\widetilde{w}$.

If conditions (1.3) and (1.6) both hold, we have a result that looks very much like Theorem 1.1.

Theorem 1.5. Let $w \in \mathcal{S}(\mathcal{U}, \mathcal{Y})$, let $t_{0} \in \mathbb{T}$ and assume that conditions (1.3) and (1.6) hold. Then the limits

$$
w_{0}:=\lim _{z \rightarrow t_{0}} w(z) \quad \text { and } \quad w_{1}:=\mathrm{w}_{-} \lim _{z \rightarrow t_{0}} w^{\prime}(z)
$$

exist along with the weak limits (1.4) and (1.7). Moreover, $w_{0}$ is unitary and $D^{w}\left(t_{0}\right)=t_{0} w_{1} w_{0}^{*}=w_{0} \widetilde{D}^{w}\left(t_{0}\right) w_{0}^{*} \geq 0$.

Let us highlight the main distinctions between Theorems 1.2 and 1.5. The sole assumption (1.3) about $D^{w}(z)$ guarantees the strong nontangential convergence of $w(z)^{*}$ (not the uniform nor even the strong convergence of $w(z)$ ) to a coisometric (not unitary, in general) operator $w_{0}$. It also implies the weak convergence of $w^{\prime}(z)$ and of $\widetilde{D}^{w}(z)$ only on the subspace $\overline{\operatorname{Ran} w_{0}^{*}} \subset \mathcal{U}$ rather than on all of $\mathcal{U}$. On the other hand, if $\operatorname{dim} \mathcal{Y}=\operatorname{dim} \mathcal{U}<\infty$, conditions (1.3) and (1.6) are equivalent; and in this case, one of them can be dropped in the formulation of Theorem 1.5.

In [4], the Carathéodory-Julia theorem was extended in a different direction: condition (1.1) was replaced by its higher order analogue

$$
\liminf _{z \rightarrow t_{0}} \frac{\partial^{2 n}}{\partial z^{n} \partial \bar{z}^{n}} \frac{1-|w(z)|^{2}}{1-|z|^{2}}<\infty
$$

with $n \geq 0$ a fixed integer. The significance of this condition for boundary interpolation theory was justified in [6]; equivalent reformulations of (1.9) in terms of nontangential boundary limits of $w$ and its derivatives at $t_{0}$ were given in [5]. In this paper, we focus on operator-valued analogues of condition (1.9),

$$
\liminf _{z \rightarrow t_{0}}\left\langle\frac{\partial^{2 n} D^{w}(z)}{\partial z^{n} \partial \bar{z}^{n}} y, y\right\rangle_{\mathcal{Y}}<\infty \quad \text { and } \quad \liminf _{z \rightarrow t_{0}}\left\langle\frac{\partial^{2 n} \widetilde{D}^{w}(z)}{\partial z^{n} \partial \bar{z}^{n}} u, u\right\rangle_{\mathcal{U}}<\infty
$$

holding for every $y \in \mathcal{Y}$ and every $u \in \mathcal{U}$, respectively. These conditions are the higher order counterparts of (1.3) and (1.6). In Section 2 we discuss the consequences of conditions (1.10) in terms of the boundary behavior of $D^{w}$ and $\widetilde{D}^{w}$ and of angular boundary derivatives of $w$. The case where both conditions in (1.10) hold is covered by Theorem 2.6, the higher order analogue of Theorem 1.5. In Section 3, we consider a more general left-tangential version of the first condition in (1.10), where $y$ is replaced by $\mathbf{a}(z) g$ for a fixed $\mathcal{L}(\mathcal{G}, \mathcal{Y})$-valued function 
a and a vector $g$ running through a given Hilbert space $\mathcal{G}$. The corresponding Carathéodory-Julia type theorem (Theorem 3.1) is the main result of the paper. To prove it, we follow D. Sarason's approach $[13,14]$, making extensive use of the de Branges-Rovnyak space $H^{w}$ associated with a Schur class function $w$. The definition and some basic properties of the space $H^{w}$ are recalled in Section 4. In Sections 5 and 6, we construct certain boundary kernels which exist and reproduce boundary derivatives of functions from the de Branges-Rovnyak space $H^{w}$ under Carathéodory-Julia type conditions. Using the reproducing properties of the boundary kernels, we give a straightforward proof of the main result in Section 7. Finally, in Section 8, we characterize the Carathéodory-Julia condition in terms of boundary behavior of functions from $H^{w}$.

\section{The higher order analogues}

We start with a result which justifies in particular, the uniform convergence of the limit $w_{0}$ in (1.8). We write

$$
\begin{gathered}
\mathcal{U}_{t_{0}, \varepsilon}:=\left\{z \in \mathbb{D}: 0<\left|z-t_{0}\right|<\varepsilon\right\}, \\
\Gamma_{t_{0}, \alpha, \varepsilon}:=\left\{z \in \mathcal{U}_{t_{0}, \varepsilon}:\left|\arg \left(z-t_{0}\right)\right|<\alpha \in(0, \pi / 2)\right\}
\end{gathered}
$$

respectively for a deleted and for a nontangential deleted neighborhood of $t_{0} \in \mathbb{T}$.

Lemma 2.1. Let $t_{0} \in \mathbb{T}$, let $f$ be an $\mathcal{L}(\mathcal{U}, \mathcal{Y})$-valued function analytic on $\mathcal{U}_{t_{0}, \varepsilon}$ and assume that $f_{n}(z):=\frac{f^{(n)}(z)}{n !}$ is bounded on $\Gamma_{t_{0}, \alpha, \varepsilon}$ for every $\alpha \in[0, \pi / 2)$ :

$$
\left\|f_{n}(z)\right\| \leq \gamma_{\alpha} \quad\left(z \in \Gamma_{t_{0}, \alpha, \varepsilon}\right) .
$$

Then the uniform limits $f_{j}\left(t_{0}\right)=\lim _{z \rightarrow t_{0}} f^{(j)}(z) / j$ ! exist for $j=0, \ldots, n-1$. In particular, the statement holds if the weak limit $\underset{z}{\mathrm{w}-\lim _{z \rightarrow t_{0}}} f_{n}(z)$ exists.

Proof. We start with the Taylor representation

$$
f_{j}(z)=\sum_{i=0}^{n-1-j} \frac{f_{j}^{(i)}(\omega)}{i !}(z-\omega)^{i}+\int_{\omega}^{z} \frac{f_{j}^{(n-j)}(\zeta)}{(n-1-j) !}(z-\zeta)^{n-1-j} d \zeta
$$

for $f_{j}(z)=f^{(j)}(z) / j$ ! at a point $\omega \in \mathcal{U}_{t_{0}, \varepsilon}$ and pick $\alpha$ such that $\omega \in \Gamma_{t_{0}, \alpha, \varepsilon}$. Since

$$
\frac{f_{j}^{(i)}(z)}{i !}=\frac{f^{(j+i)}(z)}{i ! j !}=\frac{(j+i) !}{i ! j !} f_{j+1}(z)=\left(\begin{array}{c}
j+i \\
j
\end{array}\right) f_{j+1}(z),
$$


the latter representation can be written as

$$
f_{j}(z)=\sum_{i=0}^{n-1-j}\left(\begin{array}{c}
j+i \\
j
\end{array}\right) f_{j+i}(\omega)(z-\omega)^{i}+n\left(\begin{array}{c}
n-1 \\
j
\end{array}\right) \int_{\omega}^{z} f_{n}(\zeta)(z-\zeta)^{n-1-j} d \zeta .
$$

The integral in (2.4) does not depend on the path of integration between $\omega$ and $z$. For brevity, we write (integrating along a rectifiable Jordan curve inside $\Gamma_{t_{0}, \alpha, \varepsilon}$ connecting $z$ and $\omega$ )

$$
G_{\omega, z}:=\int_{\omega}^{z} f_{n}(\zeta)(z-\zeta)^{n-1-j} d \zeta
$$

By (2.3), $\left\|G_{\omega, z_{1}}-G_{\omega, z_{2}}\right\| \leq \gamma_{\alpha} 2^{n-1-j}\left|z_{1}-z_{2}\right|$; and thus, for a fixed $\omega$, the family $\left\{G_{\omega, z}\right\}_{z \in \Gamma_{t_{0}, \alpha, \varepsilon}}$ is fundamental in the uniform operator topology. By the completeness of $\mathcal{L}(\mathcal{U}, \mathcal{Y})$, the uniform limit

$$
\lim _{\Gamma_{t_{0}, \alpha, \varepsilon} \ni z \rightarrow t_{0}} G_{\omega, z}=: G_{\omega, t_{0}}=\int_{\omega}^{t_{0}} f_{n}(\zeta)\left(t_{0}-\zeta\right)^{n-1-j} d \zeta
$$

exists; and, since it exists for every $\alpha \in[0, \pi / 2)$, it can be replaced by the nontangential limit: $G_{\omega, t_{0}}=\lim _{z \breve{\rightarrow} t_{0}} G_{\omega, z}$. Now let $z$ to tend to $t_{0}$ nontangentially in (2.4) (for $\omega$ fixed) to conclude that the uniform limits

$$
f_{j}\left(t_{0}\right)=\lim _{z \rightarrow t_{0}} f_{j}(z)=\sum_{i=0}^{n-1-j}\left(\begin{array}{c}
j+i \\
j
\end{array}\right) f_{j+i}(\omega)\left(t_{0}-\omega\right)^{i}+n\left(\begin{array}{c}
n-1 \\
j
\end{array}\right) G_{\omega, t_{0}}
$$

exist for $j=0, \ldots, n-1$. To complete the proof of the lemma, it remains only to note that weak convergence implies boundedness in norm, i.e., that the existence of the weak limit $f_{n}\left(t_{0}\right):=\underset{z-\lim }{\rightarrow} f_{0} f_{n}(z)$ implies (2.3).

To formulate higher order Carathéodory-Julia theorems, we first introduce some notation. With an operator valued function $w$ (not necessarily in the Schur class) analytic at $z \in \mathbb{D}$, we associate the operator block matrices

$$
\begin{aligned}
& \mathbf{P}_{n}^{w}(z):=\left[\frac{1}{i ! j !} \frac{\partial^{i+j}}{\partial z^{i} \partial \bar{z}^{j}} \frac{\mathrm{I}_{\mathcal{Y}}-w(z) w(z)^{*}}{1-|z|^{2}}\right]_{i, j=0}^{n}, \\
& \widetilde{\mathbf{P}}_{n}^{w}(z):=\left[\frac{1}{i ! j !} \frac{\partial^{i+j}}{\partial \bar{z}^{i} \partial z^{j}} \frac{\mathrm{I}_{\mathcal{U}}-w(z)^{*} w(z)}{1-|z|^{2}}\right]_{i, j=0}^{n},
\end{aligned}
$$

which we refer to as to Schwarz-Pick matrices. We extend definitions (2.5) and (2.6) to boundary points as follows: given a point $t_{0} \in \mathbb{T}$, the boundary Schwarz-Pick matrices are

$$
\mathbf{P}_{n}^{w}\left(t_{0}\right)=\mathrm{w}_{z} \lim _{z \rightarrow t_{0}} \mathbf{P}_{n}^{w}(z) \quad \text { and } \quad \widetilde{\mathbf{P}}_{n}^{w}\left(t_{0}\right)=\mathrm{w}_{z} \lim _{z \rightarrow t_{0}} \widetilde{\mathbf{P}}_{n}^{w}(z)
$$


provided the limits in (2.7) exist. A well-known property of Schur functions $w \in \mathcal{S}(\mathcal{U}, \mathcal{Y})$ is that $\mathbf{P}_{n}^{w}(z)$ and $\widetilde{\mathbf{P}}_{n}^{w}(z)$ are positive semidefinite for every $n \geq 0$ and $z \in \mathbb{D}$; therefore, the boundary Schwarz-Pick matrices (once they exist) are positive semidefinite as well. We also associate with $w$ the Toeplitz and the Hankel operator block matrices

(2.8) $\mathbf{T}_{n}^{w}(z)=\left[\begin{array}{cccc}w_{0}(z) & 0 & \ldots & 0 \\ w_{1}(z) & \ddots & \ddots & \vdots \\ \vdots & \ddots & \ddots & 0 \\ w_{n}(z) & \ldots & w_{1}(z) & w_{0}(z)\end{array}\right] \quad$ and $\quad \mathbf{H}_{n}^{w}(z)=\left[w_{i+j+1}(z)\right]_{i, j=0}^{n}$,

where $w_{j}(z):=w^{(j)}(z) / j$ ! stands for the $j$-th Taylor coefficient of $w$ at $z$. If $w$ is analytic on $\mathbb{D}$ and the nontangential boundary limits

$$
w_{j}\left(t_{0}\right):=\mathrm{w}_{z \rightarrow \lim _{0}} w_{j}(z)=\mathrm{w}-\lim \underset{z \rightarrow t_{0}}{ } \frac{w^{(j)}(z)}{j !}
$$

exist at a boundary point $t_{0} \in \mathbb{T}$, we extend definitions (2.8) to $t_{0}$ by

$$
\begin{aligned}
\mathbf{T}_{n}^{w}\left(t_{0}\right) & =\underset{z}{\mathrm{w}-\lim _{z \rightarrow t_{0}}} \mathbf{T}_{n}^{w}(z)=\left[\begin{array}{ccc}
w_{0}\left(t_{0}\right) & & 0 \\
\vdots & \ddots & \\
w_{n}\left(t_{0}\right) & \ldots & w_{0}\left(t_{0}\right)
\end{array}\right], \\
\mathbf{H}_{n}^{w}\left(t_{0}\right) & =\underset{z}{\mathrm{w}-\lim _{z \rightarrow t_{0}}} \mathbf{H}_{n}^{w}(z)=\left[w_{i+j+1}\left(t_{0}\right)\right]_{i, j=0}^{n}
\end{aligned}
$$

and call $w_{j}\left(t_{0}\right)$ the $j$-th angular derivative of $w$ at $t_{0}$. Furthermore, if the limits (2.9) exist for $j=0, \ldots, n$, we introduce the (operator valued) polynomials $p_{j}^{w}(z)=$ $\sum_{\ell=0}^{j}\left(z-t_{0}\right)^{\ell} w_{\ell}\left(t_{0}\right)^{*}$ and

$$
p_{j}^{w \sharp}(z):=z^{j} p_{j}^{w}(1 / \bar{z})^{*}=\sum_{\ell=0}^{j} z^{j-\ell}\left(1-z \bar{t}_{0}\right)^{\ell} w_{\ell}\left(t_{0}\right)^{*}
$$

for $j=0,1, \ldots, n$. We set

$$
p_{j, i}^{w \sharp}(z):=\frac{1}{i !} \frac{d^{i}}{d z^{i}} p_{j}^{w \sharp}(z) \quad \text { for } 0 \leq i \leq j \leq n
$$

and introduce the polynomial upper triangular block matrix

$$
\mathbf{U}_{n}^{w}(z)=\left[\begin{array}{cccc}
p_{0,0}^{w \sharp}(z) & p_{1,1}^{w \sharp}(z) & \ldots & p_{n, n}^{w \sharp}(z) \\
0 & p_{1,0}^{w \sharp}(z) & \ldots & p_{n, n-1}^{w \sharp}(z) \\
\vdots & \ddots & \ddots & \vdots \\
0 & \cdots & 0 & p_{n, 0}^{w \sharp}(z)
\end{array}\right]
$$


with the block entries $\mathbf{U}_{i j}^{w}(z)= \begin{cases}p_{j, j-i}^{w \sharp}(z) & \text { if } j \geq i, \\ 0, & \text { if } j<i .\end{cases}$

The next theorem is the higher order analogue of Theorem 1.2.

Theorem 2.2. Let $w \in \mathcal{S}(\mathcal{U}, \mathcal{Y}), t_{0} \in \mathbb{T}$ and $n \in \mathbb{Z}_{+}$and assume that

$$
\liminf _{z \rightarrow t_{0}}\left\langle\frac{\partial^{2 n}}{\partial z^{n} \partial \bar{z}^{n}} \frac{\mathrm{I}_{\mathcal{Y}}-w(z) w(z)^{*}}{1-|z|^{2}} y, y\right\rangle_{\mathcal{Y}}<\infty \quad \text { for every } y \in \mathcal{Y} .
$$

Then the following statements hold.

(1) The boundary Schwarz-Pick matrix $\mathbf{P}_{n}^{w}\left(t_{0}\right)$ defined in (2.7) exists.

(2) The strong limits

$$
w_{j}\left(t_{0}\right)^{*}:=\mathrm{s}_{z \rightarrow t_{0}} \mathrm{lim}_{j}(z)^{*} \quad(j=0, \ldots, n)
$$

exist (by Lemma 2.1, the first $n$ of them converge in norm) and the operator $w_{0}\left(t_{0}\right)$ is coisometric.

Let $\mathbf{U}_{n}^{w}(z)$ be the upper triangular polynomial matrix associated with the limits $w_{0}\left(t_{0}\right), \ldots, w_{n}\left(t_{0}\right)$ via formula (2.14), let $\mathbf{H}_{n}^{w}(z)$ be as in (2.11), and let $\mathbf{M}$ be the diagonal matrix defined as

$$
\mathbf{M}=\operatorname{diag}\left\{t_{0} \mathrm{I}_{\mathcal{Y}},-t_{0}^{2} \mathrm{I}_{\mathcal{Y}}, \ldots,(-1)^{n} t_{0}^{n+1} \mathrm{I}_{\mathcal{Y}}\right\} .
$$

(3) The weak limits

$$
\begin{aligned}
\mathbf{Q}_{n}^{w}\left(t_{0}\right) & :=\mathrm{w}_{z}-\lim _{z \rightarrow t_{0}} \mathbf{U}_{n}^{w}(z)^{*} \widetilde{\mathbf{P}}_{n}^{w}(z) \mathbf{U}_{n}^{w}(z), \\
\mathbb{P}_{n}^{w}\left(t_{0}\right) & :=\mathrm{w}_{z \rightarrow t_{0}} \mathbf{H}_{n}(z) \mathbf{U}_{n}^{w}(z) \mathbf{M}
\end{aligned}
$$

exist and, moreover, $\mathbf{P}_{n}^{w}\left(t_{0}\right)=\mathbb{P}_{n}^{w}\left(t_{0}\right)=\mathbf{M}^{*} \mathbf{Q}_{n}^{w}\left(t_{0}\right) \mathbf{M} \geq 0$.

The proof is given later (see Remark 3.2 below). Here we make several remarks and point out some corollaries of Theorem 2.2.

Remark 2.3. When $n=0$, Theorem 2.2 reduces to Theorem 1.2. This is readily seen once we observe that $\mathbf{P}_{0}^{w}(z)=D^{w}(z), \widetilde{\mathbf{P}}_{0}^{w}(z)=\widetilde{D}^{w}(z)$,

$$
\mathbf{H}_{0}^{w}(z)=w^{\prime}(z), \quad \mathbf{U}_{0}^{w}(z)=p_{0,0}^{w \sharp}(z) \equiv w_{0}\left(t_{0}\right)^{*}, \quad \mathbf{M}=t_{0} \mathbf{I}_{\mathcal{Y}} .
$$

Remark 2.4. Making use of the function $\widetilde{w}(z):=w(\bar{z})^{*} \in \mathcal{S}(\mathcal{Y}, \mathcal{U})$, as has been explained in Remark 1.4, one can easily derive a dual version of 
Theorem 2.2 (the higher order analogue of Theorem 1.3) concerning consequences of the condition

$$
\liminf _{z \rightarrow t_{0}}\left\langle\frac{\partial^{2 n}}{\partial z^{n} \partial \bar{z}^{n}} \frac{\mathrm{I}_{\mathcal{U}}-w(z)^{*} w(z)}{1-|z|^{2}} u, u\right\rangle_{\mathcal{U}}<\infty \quad \text { for every } u \in \mathcal{U}
$$

We omit the detailed formulation of this result and simply mention that (2.20) guarantees the existence of the boundary Schwarz-Pick matrix $\widetilde{\mathbf{P}}_{n}^{w}\left(t_{0}\right)$ (see the second formula in (2.7)) and, in particular, implies (1.6).

Now we pass to the case where conditions (2.15) and (2.20) both hold. The next proposition shows that one of these two conditions can be slightly relaxed.

Proposition 2.5. Let $w \in \mathcal{S}(\mathcal{U}, \mathcal{Y}), t_{0} \in \mathbb{T}$ and assume that condition (2.15) holds, so that the limit $w_{0}\left(t_{0}\right)=\mathrm{w}_{z \rightarrow t_{0}} w(z)$ exists and is coisometric (by Theorem 2.2). The following statements are equivalent:

(1) $w_{0}\left(t_{0}\right)$ is unitary; (2) condition (1.6) holds; (3) condition (2.20) holds. In case $\operatorname{dim} \mathcal{U}=\operatorname{dim} \mathcal{Y}<\infty$, statements (1)-(3) follow from (2.15).

Proof. Assume that condition (2.15) holds and that $w_{0}\left(t_{0}\right)$ is unitary. Letting $z=t_{0}$ in (2.12) gives $p_{j, 0}^{w \sharp}\left(t_{0}\right)=t_{0}^{j} w_{0}\left(t_{0}\right)^{*}$, and therefore all the diagonal blocks in the upper triangular matrix $\mathbf{U}_{n}^{w}\left(t_{0}\right)$ are unitary. Therefore, $\mathbf{U}_{n}^{w}\left(t_{0}\right)$ is boundedly invertible. Since the $\mathbf{U}_{n}^{w}(z)$ converges in norm (since $\mathbf{U}_{n}^{w}(z)$ is a polynomial) to a boundedly invertible operator $\mathbf{U}_{n}^{w}\left(t_{0}\right)$, the limit $\underset{z}{\mathrm{w}} \lim _{z \rightarrow t_{0}} \widetilde{\mathbf{P}}_{n}^{w}(z)$ also exists. In particular, the weak limit of the rightmost diagonal block in $\widetilde{\mathbf{P}}_{n}^{w}(z)$ exists, which clearly implies (2.20). This completes the proof of implication (1) $\Rightarrow(3)$. Implication $(3) \Rightarrow(2)$ holds true independent of condition (2.15) (see Remark 2.4). Implication $(2) \Rightarrow(1)$ follows by Theorem 1.5. Finally, condition (2.15) implies that $w_{0}\left(t_{0}\right)$ is coisometric; and if $\operatorname{dim} \mathcal{U}=\operatorname{dim} \mathcal{Y}<\infty$, then (2) follows.

The next theorem is the higher order analogue of Theorem 1.5. Condition (1.6) in its formulation can be equivalently replaced by condition (2.20) or by the assumption that the boundary limit $w_{0}\left(t_{0}\right)$ is unitary.

Theorem 2.6. Let $w \in \mathcal{S}(\mathcal{U}, \mathcal{Y}), t_{0} \in \mathbb{T}, n \in \mathbb{Z}_{+}$and assume that conditions (1.6) and (2.15) hold. Then the following statements hold.

(1) The matrices $\mathbf{P}_{n}^{w}\left(t_{0}\right)$ and $\widetilde{\mathbf{P}}_{n}^{w}\left(t_{0}\right)$ defined via the weak limits (2.7) exist.

(2) There exist the uniform limits

$$
w_{j}\left(t_{0}\right):=\lim _{z \widehat{\rightarrow} t_{0}} w_{j}(z) \quad \text { for } j=0, \ldots, 2 n
$$


and the weak limit $w_{2 n+1}\left(t_{0}\right):=\underset{z \rightarrow t_{0}}{\mathrm{w}-\lim _{2 n+1}} w_{2 n}$. Moreover, $w_{0}\left(t_{0}\right)$ is unitary and

$$
\mathbf{P}_{n}^{w}\left(t_{0}\right)=\mathbf{H}_{n}^{w}\left(t_{0}\right) \boldsymbol{\Psi} \mathbf{T}_{n}^{w}\left(t_{0}\right)^{*}=\mathbf{T}_{n}^{w}\left(t_{0}\right) \mathbf{\Psi}^{*} \widetilde{\mathbf{P}}_{n}^{w}\left(t_{0}\right) \mathbf{\Psi} \mathbf{T}_{n}^{w}\left(t_{0}\right)^{*} \geq 0
$$

where $\mathbf{T}_{n}^{w}\left(t_{0}\right)$ and $\mathbf{H}_{n}^{w}\left(t_{0}\right)$ are given by (2.10), (2.11) and $\mathbf{\Psi}$ is the upper triangular block matrix given by

$$
\boldsymbol{\Psi}=\left[\Psi_{j \ell} \cdot \mathrm{I}_{\mathcal{Y}}\right]_{j, \ell=0}^{n}, \quad \Psi_{j \ell}= \begin{cases}0, & \text { if } j>\ell, \\
(-1)^{\ell}\left(\begin{array}{c}
\ell \\
j
\end{array}\right) t_{0}^{\ell+j+1}, & \text { if } j \leq \ell .\end{cases}
$$

Proof. We prove the theorem assuming that Theorem 2.2 is already proved. By Theorem 2.2, condition (2.15) alone guarantees the existence of $\mathbf{P}_{n}\left(t_{0}\right)$ and of the limits (2.16)-(2.19). The boundary limit $w_{0}\left(t_{0}\right)$ is unitary, by Proposition 2.5; and $\widetilde{\mathbf{P}}_{n}\left(t_{0}\right)$ exists by Remark 2.4. Since $\mathbf{U}_{n}^{w}(z)$ converges in norm to a boundedly invertible operator $\mathbf{U}_{n}^{w}\left(t_{0}\right)$, it follows from (2.19) that the weak limit $\mathbf{H}_{n}\left(t_{0}\right)$ in (2.11) exists. This implies, in particular, the existence of the weak limit $w_{2 n+1}\left(t_{0}\right)$ which, in turn, implies the existence of the uniform limits (2.21), by Lemma 2.1. Since $\mathbf{U}_{n}^{w}(z)$ is a polynomial, it converges to $\mathbf{U}_{n}^{w}\left(t_{0}\right)$ in norm as $z \rightarrow t_{0}$; therefore, the limits on the right hand sides of (2.18) and (2.19) can be evaluated as follows:

$$
\mathbf{Q}_{n}^{w}\left(t_{0}\right)=\mathbf{U}_{n}^{w}\left(t_{0}\right)^{*} \widetilde{\mathbf{P}}_{n}^{w}\left(t_{0}\right) \mathbf{U}_{n}^{w}\left(t_{0}\right) \quad \text { and } \quad \mathbb{P}_{n}^{w}\left(t_{0}\right)=\mathbf{H}_{n}^{w}\left(t_{0}\right) \mathbf{U}_{n}^{w}\left(t_{0}\right) \mathbf{M} .
$$

Next we show that

$$
\mathbf{U}_{n}^{w}\left(t_{0}\right) \mathbf{M}=\boldsymbol{\Psi} \mathbf{T}_{n}^{w}\left(t_{0}\right)^{*},
$$

where $\mathbf{M}$ is defined in (2.17). Indeed, differentiating (2.12) $k$ times and evaluating the result at $z=t_{0}$ leads us to explicit formulas for $p_{j, k}^{w \sharp}\left(t_{0}\right)$ in terms of $w_{\ell}\left(t_{0}\right)$ :

$$
p_{j, k}^{w \sharp}\left(t_{0}\right)=\sum_{\ell=0}^{j}(-1)^{\ell} t_{0}^{j-k-\ell}\left(\begin{array}{l}
j-\ell \\
j-k
\end{array}\right) w_{\ell}\left(t_{0}\right)^{*} \quad(0 \leq k \leq j \leq n),
$$

which can be written in terms of the numbers (2.23) as

$$
(-1)^{j} t_{0}^{j+1} p_{j, k}^{w \sharp}\left(t_{0}\right)=\sum_{\ell=0}^{j} \Psi_{j-k, j-\ell} w_{\ell}\left(t_{0}\right)^{*} \quad(0 \leq k \leq j \leq n) .
$$

It is readily checked from the definitions of the matrices $\mathbf{T}_{n}^{w}\left(t_{0}\right), \mathbf{U}_{n}^{w}\left(t_{0}\right), \mathbf{M}$, and $\boldsymbol{\Psi}$ that (2.26) is just the entrywise reformulation of the matrix equality (2.25). Now we combine (2.25) and (2.24) to get

$$
\mathbf{Q}_{n}^{w}\left(t_{0}\right)=\mathbf{T}_{n}^{w}\left(t_{0}\right) \boldsymbol{\Psi}^{*} \widetilde{\mathbf{P}}_{n}^{w}\left(t_{0}\right) \mathbf{\Psi} \mathbf{T}_{n}^{w}\left(t_{0}\right)^{*}, \quad \mathbb{P}_{n}^{w}\left(t_{0}\right)=\mathbf{H}_{n}^{w}\left(t_{0}\right) \mathbf{\Psi} \mathbf{T}_{n}^{w}\left(t_{0}\right)^{*},
$$

and then (2.22) follows by the last statement in Theorem 2.2. 
Specializing Theorems 2.2 and 2.6 to the matrix valued case (i.e., to the case where the coefficient spaces $\mathcal{U}$ and $\mathcal{Y}$ are finite dimensional) is of some interest. If $\operatorname{dim} \mathcal{U}=\operatorname{dim} \mathcal{Y}$, then condition (1.6) in the formulation of Theorem 2.6 is redundant (it follows from (2.15) by Proposition 2.5). Thus, for square matrix valued Schur functions, condition (2.15) alone implies the existence of the boundary SchwarzPick matrices $\mathbf{P}_{n}^{w}\left(t_{0}\right)$ and $\widetilde{\mathbf{P}}_{n}^{w}\left(t_{0}\right)$ and of boundary angular derivatives $w_{j}\left(t_{0}\right)$ for $j=0, \ldots, 2 n+1$, which then are related as in (2.22). Extracting the part relating $\mathbf{P}_{n}^{w}\left(t_{0}\right)$ with boundary angular derivatives $w_{j}\left(t_{0}\right)$ from Theorem 2.6, we get the "only if" part in the following result.

Theorem 2.7. Let $w \in \mathcal{S}(\mathcal{U}, \mathcal{Y})$ and let $\operatorname{dim} \mathcal{U}=\operatorname{dim} \mathcal{Y}<\infty$. Then condition (2.15) holds if and only if the boundary derivatives $w_{j}\left(t_{0}\right)$ exist for $j=0, \ldots, 2 n+1$ and are such that (1) $w_{0}\left(t_{0}\right)$ is unitary and (2) $\mathbf{H}_{n}^{w}\left(t_{0}\right) \mathbf{\Psi} \mathbf{T}_{n}^{w}\left(t_{0}\right)^{*} \geq 0$.

In other words, condition (2.15) not only guarantees the existence of the boundary angular derivatives $w_{j}\left(t_{0}\right)$ but forces them to be quite special. The "if" direction in Theorem 2.7 can be proved under the weaker assumptions that $w$ is analytic on $\mathbb{D}$ (not necessarily in the Schur class) and that the matrix $\mathbf{H}_{n}^{w}\left(t_{0}\right) \mathbf{\Psi} \mathbf{T}_{n}^{w}\left(t_{0}\right)^{*}$ is Hermitian; the proof is much the same as in the scalar-valued case (see [5]). Note also that Theorems 2.6 and 2.7 can be easily translated to the context of NevanlinnaHerglotz functions or Carathéodory (positive real) functions using Caley transforms relating these functions to square matrix-valued Schur-class functions. In contrast, Theorem 2.2 does not have analogues in matrix valued Nevanlinna-Herglotz or Carathéodory classes. If $\operatorname{dim} \mathcal{Y}<\operatorname{dim} \mathcal{U}<\infty$, then condition (1.6) cannot hold at all; and the statements in Theorem 2.2 are all we can get for the non-square matrix valued Schur functions. However, if the boundary angular derivatives $w_{j}\left(t_{0}\right)$ exist for $j=n+1, \ldots, 2 n+1$, then, as was observed in [3, Lemma 12.5], we still have $\mathbf{P}_{n}^{w}\left(t_{0}\right)=\mathbf{H}_{n}^{w}\left(t_{0}\right) \mathbf{\Psi} \mathbf{T}_{n}^{w}\left(t_{0}\right)^{*}$.

\section{The main result}

The main result of the paper is Theorem 3.1 below. It is a left-tangential version of Theorem 2.2 and contains the latter theorem as a particular case. This more general theorem also displays the bi-tangential nature of the operator valued CarathéodoryJulia theorem, which is not that explicit in the formulation of Theorem 2.2.

For a Schur function $w \in \mathcal{S}(\mathcal{U}, \mathcal{Y})$ and an $\mathcal{L}(\mathcal{Y}, \mathcal{G})$-valued function a $(z)$ analytic at $t_{0}$, define the tangential Schwarz-Pick matrix

$$
\mathbf{P}_{n}^{w, \mathbf{a}}(z):=\left[\mathbf{P}_{i j}^{w, \mathbf{a}}(z)\right]_{i, j=0}^{n}
$$


where

$$
\mathbf{P}_{i j}^{w, \mathbf{a}}(z)=\frac{1}{i ! j !} \frac{\partial^{i+j}}{\partial z^{i} \partial \bar{z}^{j}}\left(\mathbf{a}(z) \frac{\mathrm{I} y-w(z) w(z)^{*}}{1-|z|^{2}} \mathbf{a}(z)^{*}\right) .
$$

This matrix is related to the matrix $\mathbf{P}_{n}^{w}(z)$ introduced in (2.5) via

$$
\mathbf{P}_{n}^{w, \mathbf{a}}(z)=\mathbf{T}_{n}^{\mathbf{a}}(z) \mathbf{P}_{n}^{w}(z) \mathbf{T}_{n}^{\mathbf{a}}(z)^{*},
$$

where $\mathbf{T}_{n}^{\mathbf{a}}(z)$ is defined according to (2.10) by

$$
\mathbf{T}_{n}^{\mathbf{a}}(z)=\left[\begin{array}{ccc}
\mathbf{a}_{0}(z) & & 0 \\
\vdots & \ddots & \\
\mathbf{a}_{n}(z) & \ldots & \mathbf{a}_{0}\left(t_{0}\right)
\end{array}\right], \quad \mathbf{a}_{\ell}(z)=\frac{\mathbf{a}^{(\ell)}(z)}{\ell !}
$$

Definition (3.2) makes sense at every point $z \in \mathbb{D}$ where a is analytic; and (3.3) follows from definitions (2.5), (3.1) and (3.4) by the Leibnitz rule. The following theorem is the main result of the paper.

Theorem 3.1. Let $w \in \mathcal{S}(\mathcal{U}, \mathcal{Y}), t_{0} \in \mathbb{T}, n \in \mathbb{Z}_{+}$, let a be an $\mathcal{L}(\mathcal{Y}, \mathcal{G})$-valued function analytic in a neighborhood of $t_{0}$ and let us assume that

$$
\liminf _{z \rightarrow t_{0}}\left\langle\frac{\partial^{2 n}}{\partial z^{n} \partial \bar{z}^{n}}\left(\mathbf{a}(z) \frac{\mathrm{I} \mathcal{Y}-w(z) w(z)^{*}}{1-|z|^{2}} \mathbf{a}(z)^{*}\right) g, g\right\rangle<\infty \quad \text { for every } g \in \mathcal{G} .
$$

Then the following statements hold.

(1) The weak limit $\mathbf{P}_{n}^{w, \mathbf{a}}\left(t_{0}\right):=\underset{z}{\mathrm{w}-\lim t_{0}} \mathbf{P}_{n}^{w, \mathbf{a}}(z)$ exists.

(2) The function $\mathbf{b}(z):=\mathbf{a}(z) w(z)$ possesses the strong boundary limits

$$
\mathbf{b}_{j}\left(t_{0}\right)^{*}:=\lim _{z \widehat{\rightarrow} t_{0}} \frac{\mathbf{b}^{(j)}(z)^{*}}{j !} \text { for } j=0, \ldots, n ;
$$

and the operator $\mathbf{b}_{0}\left(t_{0}\right)$ satisfies $\mathbf{a}_{0}\left(t_{0}\right) \mathbf{a}_{0}\left(t_{0}\right)^{*}=\mathbf{b}_{0}\left(t_{0}\right) \mathbf{b}_{0}\left(t_{0}\right)^{*}$.

Let $B_{j, i}:=p_{j, i}^{\mathbf{b} \sharp}$ be the polynomials defined via formulas (2.12), (2.13) (see also the explicit formulas (5.32) and (6.1) below) and let $\mathbf{U}_{n}^{\mathbf{b}}(z)$ be the upper triangular operator valued polynomial defined via (2.14):

$$
\mathbf{U}_{n}^{\mathbf{b}}(z)=\left[\begin{array}{cccc}
B_{0,0}(z) & B_{1,1}(z) & \ldots & B_{n, n}(z) \\
0 & B_{1,0}(z) & \ldots & B_{n, n-1}(z) \\
\vdots & \ddots & \ddots & \vdots \\
0 & \ldots & 0 & B_{n, 0}(z)
\end{array}\right] .
$$

(3) The functions $\mathbf{c}_{j}(z):=w(z) B_{j}(z)$ possess the strong boundary limits

$$
\mathbf{c}_{j, i}\left(t_{0}\right):=\lim _{z \widehat{\rightarrow} t_{0}} \frac{1}{i !}\left(w(z) B_{j}(z)\right)^{(i)} \quad \text { for } 0 \leq i \leq j \leq n .
$$


(4) The weak limits

$$
\begin{aligned}
& \mathbb{P}_{n}^{w, \mathbf{a}}\left(t_{0}\right):=\underset{z}{\mathrm{w}-\lim } \underset{t_{0}}{ } \mathbf{T}^{\mathbf{a}}(z) \mathbf{H}_{n}^{w}(z) \mathbf{U}_{n}^{\mathbf{b}}(z) \mathbf{M}, \\
& \mathbf{Q}_{n}^{w}\left(t_{0}\right):=\underset{z}{\mathrm{w}-\lim } \rightarrow t_{0} \mathbf{U}_{n}^{\mathbf{b}}(z)^{*} \widetilde{\mathbf{P}}_{n}^{w}(z) \mathbf{U}_{n}^{\mathbf{b}}(z),
\end{aligned}
$$

exist, where $\mathbf{T}_{n}^{\mathbf{a}}(z)$ and $\mathbf{H}_{n}^{w}(z)$ are given by (3.4), (2.8) and $\mathbf{M}$ is defined via formula (2.17) with the identity operator $\mathrm{I}_{\mathcal{Y}}$ replaced by $\mathrm{I}_{\mathcal{G}}$. Moreover,

$$
\mathbf{P}_{n}^{w, \mathbf{a}}\left(t_{0}\right)=\mathbb{P}_{n}^{w, \mathbf{a}}\left(t_{0}\right)=\mathbf{M}^{*} \mathbf{Q}_{n}^{w}\left(t_{0}\right) \mathbf{M} \geq 0
$$

Note that statements (1)-(3) in Theorem 3.1 were proved in [3, Section 9] for matrix-valued Schur functions under the stronger (than (3.5)) assumption that $\left\|\mathbf{P}_{n n}^{w, \mathbf{a}}(z)\right\|$ is uniformly bounded in a nontangential neighborhood of $t_{0}$.

Remark 3.2. Theorem 2.2 follows from Theorem 3.1.

Indeed, if $\mathcal{G}=\mathcal{Y}$ and $\mathbf{a}(z) \equiv \mathrm{I}_{\mathcal{Y}}$ in Theorem 3.1, then condition (3.5) collapses to (2.15). Furthermore, $\mathbf{P}_{n}^{w, \mathbf{a}}(z)=\mathbf{P}_{n}^{w}(z), \mathbf{b}(z)=w(z), \mathbf{T}^{\mathbf{a}}(z) \equiv \mathrm{I}_{\mathcal{Y}^{n+1}}$; and it is readily seen that statements (1), (2) and (3) in Theorem 2.2 follow, respectively, from statements (1), (2) and (4) in Theorem 3.1.

Another special choice $\mathcal{G}=\mathbb{C}$ and $\mathbf{a}(z) \equiv y \in \mathcal{Y}$ in Theorem 3.1 gives the consequences of condition (2.15) holding just for a fixed vector $y$. We omit the precise formulation. The proof of Theorem 3.1 is presented in Section 6. The next three sections contain definitions and constructions needed for the proof.

\section{4 de Branges-Rovnyak spaces and their reproducing kernels}

In this section, we recall the definitions of the Hilbert spaces $L^{w}$ and $H^{w}[7,8,12]$ and their properties needed for the subsequent analysis. Given a separable Hilbert space $\mathcal{U}$, we denote by $L_{2}(\mathcal{U})$ the space of $\mathcal{U}$-valued measurable functions $u(t)$ with

$$
\|u\|_{L_{2}(\mathcal{U})}^{2}:=\int_{\mathbb{T}}\|u(t)\|_{\mathcal{U}}^{2} m(d t)<\infty,
$$

where $m(d t)$ stands for the normalized arc length Lebesgue measure on $\mathbb{T}$; the symbols $H_{2}^{+}(\mathcal{U})$ and $H_{2}^{-}(\mathcal{U})$ stand for the Hardy spaces of $\mathcal{U}$-valued functions with vanishing Fourier coefficients with negative (respectively, nonnegative) indices. The elements in $H_{2}^{+}(\mathcal{U})$ and $H_{2}^{-}(\mathcal{U})$ are identified with their unique analytic (resp., conjugate-analytic) continuations inside the unit disk so that $H_{2}^{+}(\mathcal{U})$ and $H_{2}^{-}(\mathcal{U})$ are identified with the Hardy spaces of the unit disk. 
Let $w: \mathbb{D} \rightarrow \mathcal{L}(\mathcal{U}, \mathcal{Y})$ be a Schur function and let $W(t):=\left[\begin{array}{cc}\mathrm{I}_{\mathcal{Y}} & w(t) \\ w(t)^{*} & \mathrm{I}_{\mathcal{U}}\end{array}\right]$. The space $L^{w}$ is the range space $W^{1 / 2}\left(L_{2}(\mathcal{Y} \oplus \mathcal{U})\right)$ endowed with the range norm. In more detail: for every element $f$ in $L^{w}$, there exists a unique $g_{f} \in L_{2}(\mathcal{Y} \oplus \mathcal{U})$ which is orthogonal to $\operatorname{Ker} W(t)$ for almost all $t \in \mathbb{T}$ and such that $f=W^{1 / 2} g_{f}$. This unique $g_{f}$ is denoted by $g_{f}:=W^{[-1 / 2]} f$, and the $L^{w}$-norm is defined by

$$
\langle f, h\rangle_{L^{w}}=\left\langle g_{f}, g_{h}\right\rangle_{L_{2}(\mathcal{Y} \oplus \mathcal{U})}=\int_{\mathbb{T}}\left\langle W(t)^{[-1 / 2]} f, W(t)^{[-1 / 2]} h\right\rangle_{\mathcal{Y} \oplus \mathcal{U}} m(d t) .
$$

Definition 4.1. A function $f=\left[\begin{array}{l}f_{+} \\ f_{-}\end{array}\right]$is said to belong to the de Branges-Rovnyak space $H^{w}$ if it belongs to $L^{w}$ and if $f_{+} \in H_{2}^{+}(\mathcal{Y})$ and $f_{-} \in H_{2}^{-}(\mathcal{U})$.

The space $H^{w}$ is a closed subspace of $L^{w}$; in what follows, $P_{H^{w}}$ denotes the orthogonal projection of $L^{w}$ onto $H^{w}$.

Recall that $H_{2}^{+}(\mathcal{Y})$ and $H_{2}^{-}(\mathcal{U})$ are reproducing kernel Hilbert spaces with reproducing kernels $k_{z}(t)=\frac{1}{1-t \bar{z}}$ and $\widetilde{k}_{z}(t)=\frac{1}{t-z}$ in the sense that

$$
\left\langle f_{+}, k_{z} y\right\rangle_{L_{2}(\mathcal{Y})}=\left\langle f_{+}(z), y\right\rangle_{\mathcal{Y}}, \quad\left\langle f_{-}, \widetilde{k}_{z} u\right\rangle_{L_{2}(\mathcal{U})}=\left\langle\frac{f_{-}(z)}{\bar{z}}, u\right\rangle_{\mathcal{U}}
$$

for every $f_{+} \in H_{2}^{+}(\mathcal{Y}), f_{-} \in H_{2}^{-}(\mathcal{U}), y \in \mathcal{Y}, u \in \mathcal{U}$ and $z \in \mathbb{D}$. We let

$$
\begin{aligned}
k_{j, z}(t) & :=\frac{1}{j !} \frac{\partial^{j}}{\partial \bar{z}^{j}} k_{z}(t)=\frac{t^{j}}{(1-t \bar{z})^{j+1}}, \\
\widetilde{k}_{j, z}(t) & :=\frac{1}{j !} \frac{\partial^{j}}{\partial z^{j}} \widetilde{k}_{z}(t)=\frac{1}{(t-z)^{j+1}} .
\end{aligned}
$$

Now we introduce the operator-valued functions

$$
\begin{aligned}
& K_{z}(t)=k_{z}(t) \cdot\left[\begin{array}{cc}
\mathrm{I}_{\mathcal{Y}} & w(t) \\
w(t)^{*} & \mathrm{I}_{\mathcal{U}}
\end{array}\right]\left[\begin{array}{c}
\mathrm{I}_{\mathcal{Y}} \\
-w(z)^{*}
\end{array}\right], \\
& \widetilde{K}_{z}(t)=\widetilde{k}_{z}(t) \cdot\left[\begin{array}{cc}
\mathrm{I}_{\mathcal{Y}} & w(t) \\
w(t)^{*} & \mathrm{I}_{\mathcal{U}}
\end{array}\right]\left[\begin{array}{c}
-w(z) \\
\mathrm{I}_{\mathcal{U}}
\end{array}\right]
\end{aligned}
$$

defined for $z \in \mathbb{D}$ and $t \in \mathbb{T}$ and, more generally, the vector-valued functions

$$
K_{z}^{(j)}(t)=\frac{1}{j !} \frac{\partial^{j}}{\partial \bar{z}^{j}} K_{z}(t) \quad \text { and } \quad \widetilde{K}_{z}^{(j)}(t)=\frac{1}{j !} \frac{\partial^{j}}{\partial z^{j}} \widetilde{K}_{z}(t)
$$

for $j \in \mathbb{Z}_{+}$. The next lemma displays reproducing properties of $K_{z}$ and $\widetilde{K}_{z}$. The straightforward proof is based on relations (4.2), formulas (4.3)-(4.6) and the definition (4.1) of the inner product in $L^{w}$ (see [4, Lemma 2.2] for the proof). 
Lemma 4.2. For every $j \in \mathbb{Z}_{+}, z \in \mathbb{D}, y \in \mathcal{Y}$, and $u \in \mathcal{U}$, the functions $K_{z}^{(j)} y$ and $\widetilde{K}_{z}^{(j)}$ u belong to $H^{w}$. Furthermore, for every $f=\left[\begin{array}{l}f_{+} \\ f_{-}\end{array}\right] \in H^{w}$,

$$
\begin{aligned}
& \left\langle f, K_{z}^{(j)} y\right\rangle_{H^{w}}=\frac{1}{j !}\left\langle\frac{d^{j}}{d z^{j}} f_{+}(z), y\right\rangle_{\mathcal{Y}}, \\
& \left\langle f, \widetilde{K}_{z}^{(j)} u\right\rangle_{H^{w}}=\frac{1}{j !}\left\langle\frac{d^{j}}{d \bar{z}^{j}}\left(\frac{f_{-}(z)}{\bar{z}}\right), u\right\rangle_{\mathcal{U}} .
\end{aligned}
$$

We conclude this section with three more lemmas. The proofs are much the same as in the scalar case (see Lemmas 2.6, 2.7 and 2.8 in [4]) and are omitted. The first lemma gives a convenient representation of the kernels $K_{z}^{(j)}$ and $\widetilde{K}_{z}^{(j)}$ as orthogonal projections of certain simple elements in $L^{w}$ onto $H^{w}$.

Lemma 4.3. Let $w \in \mathcal{S}(\mathcal{U}, \mathcal{Y})$, let $z \in \mathbb{D}, j \in \mathbb{Z}_{+}$and let $K_{z}^{(j)}$ and $\widetilde{K}_{z}^{(j)}$ be the functions defined in (4.7). Then for every pair of vectors $y \in \mathcal{Y}$ and $u \in \mathcal{U}$,

$$
\begin{aligned}
& K_{z}^{(j)} y=P_{H^{w}}\left(\left[\begin{array}{cc}
\mathrm{I}_{\mathcal{Y}} & w \\
w^{*} & \mathrm{I}_{\mathcal{U}}
\end{array}\right]\left[\begin{array}{c}
k_{j, z} \cdot y \\
0
\end{array}\right]\right), \\
& \widetilde{K}_{z}^{(j)} u=P_{H^{w}}\left(\left[\begin{array}{cc}
\mathrm{I}_{\mathcal{Y}} & w \\
w^{*} & \mathrm{I}_{\mathcal{U}}
\end{array}\right]\left[\begin{array}{c}
0 \\
\widetilde{k}_{j, z} \cdot u
\end{array}\right]\right) .
\end{aligned}
$$

Lemma 4.4. Let $P_{H^{w}}$ be the orthogonal projection of $L^{w}$ onto $H^{w}$.

(1) If $g_{1} \in L_{2}(\mathcal{Y})$ and $g_{2} \in H_{2}^{+}(\mathcal{U})$, then

$$
P_{H^{w}}\left[\begin{array}{ll}
\mathrm{I}_{\mathcal{Y}} & w \\
w^{*} & \mathrm{I}_{\mathcal{U}}
\end{array}\right]\left[\begin{array}{l}
g_{1} \\
g_{2}
\end{array}\right]=P_{H^{w}}\left[\begin{array}{cc}
\mathrm{I}_{\mathcal{Y}} & w \\
w^{*} & \mathrm{I}_{\mathcal{U}}
\end{array}\right]\left[\begin{array}{c}
g_{1} \\
0
\end{array}\right] .
$$

(2) If $g_{1} \in H_{2}^{-}(\mathcal{Y})$ and $g_{2} \in L_{2}(\mathcal{U})$, then

$$
P_{H^{w}}\left[\begin{array}{cc}
\mathrm{I}_{\mathcal{Y}} & w \\
w^{*} & \mathrm{I}_{\mathcal{U}}
\end{array}\right]\left[\begin{array}{l}
g_{1} \\
g_{2}
\end{array}\right]=P_{H^{w}}\left[\begin{array}{cc}
\mathrm{I}_{\mathcal{Y}} & w \\
w^{*} & \mathrm{I}_{\mathcal{U}}
\end{array}\right]\left[\begin{array}{c}
0 \\
g_{2}
\end{array}\right] .
$$

Lemma 4.5. Let $w \in \mathcal{S}(\mathcal{U}, \mathcal{Y})$ and $h \in L^{w}$. Then for every $t_{0} \in \mathbb{T}, z \in \mathbb{D}$ and $n \in \mathbb{N}$, the functions

$$
h_{z}(t)=\left(\frac{1-t \bar{t}_{0}}{1-t \bar{z}}\right)^{n} h(t) \quad \text { and } \quad \widetilde{h}_{z}(t)=\left(\frac{t-t_{0}}{t-z}\right)^{n} h(t)
$$

belong to $L^{w}$ and tend to $h$ in norm of $L^{w}$ as $z \widehat{\rightarrow} t_{0}$ :

$$
\lim _{z \rightarrow t_{0}}\left\|h_{z}-h\right\|_{L^{w}}=0 \quad \text { and } \quad \lim _{z \rightarrow t_{0}}\left\|\widetilde{h}_{z}-h\right\|_{L^{w}}=0 .
$$




\section{The kernels $L_{z}^{(j)}$ and their boundary analogues}

For a Schur function $w \in \mathcal{S}(\mathcal{U}, \mathcal{Y})$ and an $\mathcal{L}(\mathcal{Y}, \mathcal{G})$-valued function a analytic at $t_{0}$, let us introduce the kernels

$$
L_{z}^{(j)}(t)=\frac{1}{j !} \frac{\partial^{j}}{\partial \bar{z}^{j}}\left(K_{z}(t) \mathbf{a}(z)^{*}\right) \quad(j \geq 0),
$$

where $K_{z}$ is defined as in (4.5). By the Leibnitz rule and the definition (4.7) of $K_{z}^{(j)}$,

$$
L_{z}^{(j)}(t)=\sum_{\ell=0}^{j} K_{z}^{(\ell)}(t) \mathbf{a}_{j-\ell}(z)^{*}
$$

On the other hand, letting $\mathbf{b}(z):=\mathbf{a}(z) w(z)$, we get from (4.5)

$$
K_{z}(t) \mathbf{a}(z)^{*}=\left[\begin{array}{cc}
\mathrm{I}_{\mathcal{Y}} & w(t) \\
w(t)^{*} & \mathrm{I}_{\mathcal{U}}
\end{array}\right]\left[\begin{array}{r}
\mathbf{a}(z)^{*} \\
-\mathbf{b}(z)^{*}
\end{array}\right] \cdot k_{z}(t) ;
$$

and another application of the Leibnitz rule leads us to

$$
L_{z}^{(j)}(t)=\left[\begin{array}{cc}
\mathrm{I}_{\mathcal{Y}} & w(t) \\
w(t)^{*} & \mathrm{I}_{\mathcal{U}}
\end{array}\right] \sum_{\ell=0}^{j}\left[\begin{array}{r}
\mathbf{a}_{j-\ell}(z)^{*} \\
-\mathbf{b}_{j-\ell}(z)^{*}
\end{array}\right] k_{\ell, z}(t),
$$

where $\mathbf{a}_{\ell}(z)=\mathbf{a}^{(\ell)}(z) / \ell$ ! and $\mathbf{b}_{\ell}(z)=\mathbf{b}^{(\ell)}(z) / \ell$ !. Formula (5.3) defines $L_{z}^{(j)}$ only on the unit circle. The analytic (conjugate-analytic) continuations of its top (bottom) component inside the unit disk are given by

$$
\begin{aligned}
& L_{z,+}^{(j)}(\zeta)=\sum_{\ell=0}^{j}\left(\mathbf{a}_{j-\ell}(z)^{*}-w(\zeta) \mathbf{b}_{j-\ell}(z)^{*}\right) \cdot k_{\ell, z}(\zeta), \\
& L_{z,-}^{(j)}(\zeta)=\bar{\zeta} \cdot \sum_{\ell=0}^{j}\left(w(\zeta)^{*} \mathbf{a}_{j-\ell}(z)^{*}-\mathbf{b}_{j-\ell}(z)^{*}\right) \widetilde{k}_{\ell, z}(\zeta)^{*}
\end{aligned}
$$

By definition (5.1), the kernels $L_{z}^{(j)}$ inherit the reproducing property (4.8) of $K_{z}^{(j)}$.

Lemma 5.1. For every $j \in \mathbb{Z}_{+}, g \in \mathcal{G}$ and $z \in \mathbb{D}$ at which a is analytic, the function $L_{z}^{(j)} g$ belongs to $H^{w}$; furthermore, for every $f=\left[\begin{array}{l}f_{+} \\ f_{-}\end{array}\right] \in H^{w}$,

$$
\left\langle f, L_{z}^{(j)} g\right\rangle_{H^{w}}=\frac{1}{j !}\left\langle\frac{d^{j}}{d z^{j}}\left(\mathbf{a}(z) f_{+}(z)\right), g\right\rangle_{\mathcal{G}}
$$


Proof. By Lemma 4.2, $K_{z}^{(\ell)} y_{\ell}$ belongs to $H^{w}$ for every $y_{\ell} \in \mathcal{Y}$. Choosing $y_{\ell}=\mathbf{a}_{j-\ell}(z)^{*} g$ we get the first statement of the lemma from (5.2). The rest follows from (4.8) and (5.1):

$$
\begin{aligned}
\left\langle f, L_{z}^{(j)} g\right\rangle_{H^{w}} & =\frac{1}{j !} \frac{d^{j}}{d z^{j}}\left\langle f, K_{z} \mathbf{a}(z)^{*} g\right\rangle_{H^{w}} \\
& =\frac{1}{j !} \frac{d^{j}}{d z^{j}}\left\langle f_{+}(z), \mathbf{a}(z)^{*} g\right\rangle_{\mathcal{Y}} \\
& =\frac{1}{j !} \frac{d^{j}}{d z^{j}}\left\langle\mathbf{a}(z) f_{+}(z), g\right\rangle_{\mathcal{G}} .
\end{aligned}
$$

Lemma 5.2. For every $z \in \mathbb{D}$ and $g, g^{\prime} \in \mathcal{G}$,

$$
\left\langle L_{z}^{(\ell)} g^{\prime}, L_{z}^{(j)} g\right\rangle_{H^{w}}=\left\langle\mathbf{P}_{j \ell}^{w, \mathbf{a}}(z) g^{\prime}, g\right\rangle_{\mathcal{G}},
$$

where $\mathbf{P}_{i j}^{w, \mathbf{a}}(z)$ is given in (3.2). In particular,

$$
\left\langle L_{z}^{(n)} g, L_{z}^{(n)} g\right\rangle_{H^{w}}=\left\|L_{z}^{(n)} g\right\|_{H^{w}}^{2}=\left\langle\mathbf{P}_{n n}^{w, \mathbf{a}}(z) g, g\right\rangle_{\mathcal{G}} .
$$

Proof. Upon setting $f=L_{z}^{(\ell)} g^{\prime}$ in (5.6), we get

$$
\left\langle L_{z}^{(\ell)} g^{\prime}, L_{z}^{(j)} g\right\rangle_{H^{w}}=\frac{1}{j !}\left\langle\frac{\partial^{j}}{\partial z^{j}} \mathbf{a}(z) L_{z,+}^{(\ell)}(z) g^{\prime}, g\right\rangle_{\mathcal{G}} .
$$

This coincides with (5.7), since by (5.1) and (4.5),

$$
L_{z,+}^{(\ell)}(t) g^{\prime}=\frac{1}{\ell !} \frac{\partial^{\ell}}{\partial \bar{z}^{j}}\left(K_{z,+}(t) \mathbf{a}(z)^{*} g^{\prime}\right)=\frac{1}{\ell !} \frac{\partial^{\ell}}{\partial \bar{z}^{j}}\left(\frac{\mathrm{I} \mathcal{Y}-w(t) w(z)^{*}}{1-t \bar{z}} \mathbf{a}(z)^{*}\right) g^{\prime}
$$

and, on the other hand, $\mathbf{a}(z) L_{z,+}^{(\ell)}(z)=\mathbf{P}_{n n}^{w, \mathbf{a}}(z)$, by the last equality and (3.2). Letting $\ell=j=n$ in (5.7) gives (5.8).

It turns out that if condition (3.5) holds, then the kernels $L_{z}^{(j)}$ converge to the "boundary kernels"

$$
L_{t_{0}}^{(j)}(t):=\left[\begin{array}{cc}
\mathrm{I}_{\mathcal{Y}} & w(t) \\
w(t)^{*} & \mathrm{I}_{\mathcal{U}}
\end{array}\right] \sum_{\ell=0}^{j}\left[\begin{array}{r}
\mathbf{a}_{j-\ell}\left(t_{0}\right)^{*} \\
-\mathbf{b}_{j-\ell}\left(t_{0}\right)^{*}
\end{array}\right] k_{\ell, t_{0}}(t)
$$

for $j=0, \ldots, n$ as $z \widehat{\rightarrow} t_{0} \in \mathbb{T}$, where

$$
k_{j, t_{0}}(\zeta)=\frac{\zeta^{j}}{\left(1-\zeta \bar{t}_{0}\right)^{j+1}} \quad \text { and } \quad \tilde{k}_{j, t_{0}}(\zeta)=\frac{1}{\left(\zeta-t_{0}\right)^{j+1}}
$$


are the boundary analogues of the kernels (4.3) and $\mathbf{b}_{0}\left(t_{0}\right)^{*}, \ldots, \mathbf{b}_{n}\left(t_{0}\right)^{*}$ are defined via the limits

$$
\mathbf{b}_{j}\left(t_{0}\right)^{*}:=\mathrm{s}_{z \rightarrow t_{0}} \mathbf{b}_{j}(z)^{*}=\mathrm{s}_{z \rightarrow t_{0}}\left[\frac{(\mathbf{a}(z) w(z))^{(j)}}{j !}\right]^{*} \quad \text { for } j=0, \ldots, n,
$$

whose existence is also a consequence of (3.5).

Theorem 5.3. Let $w \in \mathcal{S}(\mathcal{U}, \mathcal{Y}), t_{0} \in \mathbb{T}, n \in \mathbb{Z}_{+}$and let us assume that condition (3.5) holds for an $\mathcal{L}(\mathcal{Y}, \mathcal{G})$-valued function a analytic at $t_{0}$. Then the following statements hold.

(1) The strong limits (5.11) exist; and the functions $L_{t_{0}}^{(j)}(t)$ defined for $j=0, \ldots, n$ by formula (5.9) belong to the space $H^{w} \otimes \mathcal{G}$ in the sense that $L_{t_{0}}^{(j)} g \in H^{w}$ for every $g \in \mathcal{G}$.

(2) The kernels $L_{z}^{(j)}$ defined by (5.3) converge to $L_{t_{0}}^{(j)}$ in norm of $H^{w} \otimes \mathcal{G}$ :

$$
L_{z}^{(j)} g \stackrel{H^{w}}{\longrightarrow} L_{t_{0}}^{(j)} g \quad \text { as } z \widehat{\longrightarrow} t_{0}
$$

for $j=0, \ldots, n$ and every vector $g \in \mathcal{G}$.

(3) The nontangential weak limit

$$
\mathbf{P}_{n n}^{w, \mathbf{a}}\left(t_{0}\right):=\underset{z}{\mathrm{w}-\lim _{z \rightarrow t_{0}}} \mathbf{P}_{n n}^{w, \mathbf{a}}(z)
$$

(where $\mathbf{P}_{n n}^{w, \mathbf{a}}(z)$ is defined in (3.2)) exists; moreover, for every $g \in \mathcal{G}$,

$$
\left\langle\mathbf{P}_{n n}^{w, \mathbf{a}}\left(t_{0}\right) g, g\right\rangle=\liminf _{z \rightarrow t_{0}}\left\langle\mathbf{P}_{n n}^{w, \mathbf{a}}(z) g, g\right\rangle .
$$

Proof. According to (5.8), condition (3.5) tells us that $\liminf _{z \rightarrow t_{0}}\left\|L_{z}^{(n)} g\right\|_{H^{w}}<\infty$ for every $g \in \mathcal{G}$. Fix $g \in \mathcal{G}$ and pick a sequence $\left\{z_{i}\right\}$ of points in $\mathbb{D}$ approaching $t_{0}$ such that the sequence $\left\|L_{z_{i}}^{(n)}\right\|_{H^{w}}$ is bounded. Since every bounded set in a Hilbert space is weakly compact, there is a subsequence of $\left\{z_{i}\right\}$ (which we continue to denote by $\left\{z_{i}\right\}$ ), such that the sequence $\left\{L_{z_{i}}^{(n)} g\right\}$ converges weakly in $H^{w}$, say to $F_{g}$ :

$$
F_{g}=\left[\begin{array}{c}
F_{g,+} \\
F_{g,-}
\end{array}\right]=\mathrm{w}_{z_{i} \rightarrow t_{0}} \lim _{z_{i}}(n) g \in H^{w}
$$

Since $F_{g}$ belongs to $H^{w}$, we can use the reproducing properties (4.8), (4.9) (with $j=0$ ) to conclude that

$$
\begin{gathered}
\left\langle F_{g,+}(\zeta), y\right\rangle_{\mathcal{Y}}=\left\langle F_{g}, K_{\zeta} y\right\rangle_{H^{w}}=\lim _{z_{i} \rightarrow t_{0}}\left\langle L_{z_{i}}^{(n)} g, K_{\zeta} y\right\rangle_{H^{w}}=\lim _{z_{i} \rightarrow t_{0}}\left\langle L_{z_{i},+}^{(n)}(\zeta) g, y\right\rangle \\
\left\langle\frac{F_{g,-}(\zeta)}{\bar{\zeta}}, u\right\rangle_{\mathcal{U}}=\left\langle F_{g}, \widetilde{K}_{\zeta} u\right\rangle_{H^{w}}=\lim _{z_{i} \rightarrow t_{0}}\left\langle L_{z_{i}}^{(n)} g, \widetilde{K}_{\zeta} u\right\rangle_{H^{w}}=\lim _{z_{i} \rightarrow t_{0}}\left\langle\frac{L_{z_{i},-}^{(n)}(\zeta)}{\bar{\zeta}} g, u\right\rangle
\end{gathered}
$$


for every $\zeta \in \mathbb{D}, y \in \mathcal{Y}$, and $u \in \mathcal{U}$. Then, by the explicit formulas (5.4) and (5.5) for $L_{z_{i},+}^{(n)}$ and $L_{z_{i},-}^{(n)}$, we have

$$
\begin{aligned}
& F_{g,+}(\zeta)=\mathrm{w}-\lim _{z_{i} \rightarrow t_{0}} \sum_{\ell=0}^{n}\left(\mathbf{a}_{\ell}\left(z_{i}\right)^{*}-w(\zeta) \mathbf{b}_{\ell}\left(z_{i}\right)^{*}\right) k_{n-\ell, z_{i}}(\zeta) g \\
& \frac{F_{g,-}(\zeta)}{\bar{\zeta}}=\mathrm{w}_{z_{i} \rightarrow t_{0}} \sum_{\ell=0}\left(w(\zeta)^{*} \mathbf{a}_{\ell}\left(z_{i}\right)^{*}-\mathbf{b}_{\ell}\left(z_{i}\right)^{*}\right) \widetilde{k}_{n-\ell, z_{i}}(\zeta)^{*} g
\end{aligned}
$$

It follows from (5.17) and formula (4.4) for $\widetilde{k}_{\ell, z}$ that

$$
\begin{aligned}
\left(\bar{\zeta}-\bar{t}_{0}\right)^{n+1} \frac{F_{g,-}(\zeta)}{\bar{\zeta}} & =\mathrm{w}_{z_{i} \rightarrow t_{0}} \sum_{\ell=0}\left(w(\zeta)^{*} \mathbf{a}_{\ell}\left(z_{i}\right)^{*}-\mathbf{b}_{\ell}\left(z_{i}\right)^{*}\right)\left(\bar{\zeta}-\bar{z}_{i}\right)^{\ell} g \\
& =w(\zeta)^{*} \sum_{\ell=0}^{n} \mathbf{a}_{\ell}\left(t_{0}\right)^{*}\left(\bar{\zeta}-\bar{t}_{0}\right)^{\ell} g-\mathrm{w}_{z_{i} \rightarrow t_{0}} \sum_{\ell=0}^{n}\left(\bar{\zeta}-\bar{z}_{i}\right)^{\ell} \mathbf{b}_{\ell}\left(z_{i}\right)^{*} g .
\end{aligned}
$$

Thus, the weak limit on the right hand side of (5.18) exists for every $\zeta \in \mathbb{D}$. Since the coefficients of a (vector-valued) polynomial of degree $n$ are determined by its values at $n+1$ points and depend on these values continuously, the existence of the latter weak limit implies that the sequences $\left\{\mathbf{b}_{\ell}\left(z_{i}\right)^{*} g\right\}_{i}$ converge weakly for every $\ell \in\{0, \ldots, n\}$. We set

$$
u_{\ell}:=\mathrm{w}_{z_{i} \rightarrow t_{0}} \lim _{\ell}\left(z_{i}\right)^{*} g \quad \text { for } \ell=0, \ldots, n .
$$

Making use of (5.19), we can rewrite (5.16) and (5.17) as

$$
\begin{aligned}
& F_{g,+}(\zeta)=\sum_{\ell=0}^{n}\left(\mathbf{a}_{\ell}\left(t_{0}\right)^{*} g-w(\zeta) u_{\ell}\right) k_{n-\ell, t_{0}}(\zeta) \\
& F_{g,-}(\zeta)=\bar{\zeta} \cdot \sum_{\ell=0}^{n}\left(w(\zeta)^{*} \mathbf{a}_{\ell}\left(t_{0}\right)^{*} g-u_{\ell}\right) \widetilde{k}_{n-\ell, t_{0}}(\zeta)^{*}
\end{aligned}
$$

Setting $\zeta=t \in \mathbb{T}$ in the last two formulas and taking into account that $\bar{t} \cdot \widetilde{k}_{j, t_{0}}(t)^{*}=$ $k_{j, t_{0}}(t)$ for $t \in \mathbb{T}$, we get the following expression for $F_{g}$ :

$$
F_{g}(t)=\left[\begin{array}{c}
F_{g,+}(t) \\
F_{g,-}(t)
\end{array}\right]=\left[\begin{array}{cc}
\mathrm{I}_{\mathcal{Y}} & w(t) \\
w(t)^{*} & \mathrm{I}_{\mathcal{U}}
\end{array}\right] \sum_{\ell=0}^{n}\left[\begin{array}{c}
\mathbf{a}_{\ell}\left(t_{0}\right)^{*} g \\
-u_{\ell}
\end{array}\right] k_{n-\ell, t_{0}}(t) .
$$

Now we show that

$$
L_{z}^{(n)} g \stackrel{H^{w}}{\longrightarrow} F_{g} \quad \text { as } z \widehat{\rightarrow} t_{0} .
$$


To this end, we introduce auxiliary functions

$$
f_{z}(t)=\left[\begin{array}{cc}
\mathrm{I}_{\mathcal{Y}} & w(t) \\
w(t)^{*} & \mathrm{I}_{\mathcal{U}}
\end{array}\right] \sum_{\ell=0}^{n}\left[\begin{array}{c}
\mathbf{a}_{\ell}(z)^{*} g \\
-u_{\ell}
\end{array}\right] k_{n-\ell, z}(t)
$$

and

$$
h_{z}(t)=\left[\begin{array}{cc}
\mathrm{I}_{\mathcal{Y}} & w(t) \\
w(t)^{*} & \mathrm{I}_{\mathcal{U}}
\end{array}\right] \sum_{\ell=0}^{n}\left[\begin{array}{c}
\mathbf{a}_{\ell}\left(t_{0}\right)^{*} g \\
-u_{\ell}
\end{array}\right] k_{n-\ell, t_{0}}(t) \cdot\left(\frac{1-t \bar{t}_{0}}{1-t \bar{z}}\right)^{n+1}
$$

which belong to $L^{w}$ for every fixed $z \in \mathbb{D}$. Since $\sum_{\ell=0}^{n} u_{\ell} k_{n-\ell, z}(t)$ belongs to $H^{2}(\mathcal{U})$, it follows by formula (4.12) in Lemma 4.4 that

$$
P_{H^{w}} f_{z}=P_{H^{w}}\left[\begin{array}{cc}
\mathrm{I}_{\mathcal{Y}} & w \\
w^{*} & \mathrm{I}_{\mathcal{U}}
\end{array}\right] \sum_{\ell=0}^{n}\left[\begin{array}{c}
k_{n-\ell, z} \cdot \mathbf{a}_{\ell}(z)^{*} g \\
0
\end{array}\right] .
$$

Therefore, by (4.10) and (5.2),

$$
\begin{aligned}
P_{H^{w}} f_{z} & =\sum_{\ell=0}^{n} P_{H^{w}}\left[\begin{array}{cc}
\mathrm{I}_{\mathcal{Y}} & w \\
w^{*} & \mathrm{I}_{\mathcal{U}}
\end{array}\right]\left[\begin{array}{c}
k_{n-\ell, z} \cdot \mathbf{a}_{\ell}(z)^{*} g \\
0
\end{array}\right] \\
& =\sum_{\ell=0}^{n} K_{z}^{(n-\ell)} \mathbf{a}_{\ell}(z)^{*} g=L_{z}^{(n)} g .
\end{aligned}
$$

On the other hand, since

$$
k_{\ell, z}(t)=\frac{t^{\ell}(1-\bar{z} t)^{n-\ell}}{(1-\bar{z} t)^{n+1}} \quad \text { converges to } \frac{t^{\ell}\left(1-\bar{t}_{0} t\right)^{n-\ell}}{(1-\bar{z} t)^{n+1}}=k_{\ell, t_{0}}(t) \cdot\left(\frac{1-t \bar{t}_{0}}{1-t \bar{z}}\right)^{n+1}
$$

uniformly in $t \in \mathbb{T}$ as $z$ tends to $t_{0}$ and since $\mathbf{a}$ is analytic at $t_{0}$ so that

$$
\lim _{z \rightarrow t_{0}}\left\|\mathbf{a}_{\ell}(z)^{*} g-\mathbf{a}_{\ell}\left(t_{0}\right)^{*} g\right\|_{\mathcal{U}}=0 \quad \text { for } \ell=0,1, \ldots,
$$

it is readily seen from (5.22) and (5.23) that

$$
\lim _{z \rightarrow t_{0}}\left\|f_{z}-h_{z}\right\|_{H^{w}}=0 .
$$

Furthermore, comparing formula (5.23) for $h_{z}$ with (5.2) gives

$$
h_{z}(t)=F_{g}(t)\left(\frac{1-t \bar{t}_{0}}{1-t \bar{z}}\right)^{n+1}
$$

Setting $h=F_{g}$ in Lemma 4.5, we conclude that the function $h_{z}$ of the form (5.26) belongs to $L^{w}$ for every $z \in \mathbb{D}$ and $h_{z} \stackrel{L^{w}}{\longrightarrow} F_{g}$ as $z \widehat{\rightarrow} t_{0}$. Combining this latter convergence with (5.25) gives $f_{z} \stackrel{L^{w}}{\longrightarrow} F_{g}$ as $z \widehat{\rightarrow} t_{0}$. Since $F_{g} \in H^{w}$, we have

$$
P_{H^{w}} h_{z} \stackrel{H^{w}}{\longrightarrow} P_{H^{w}} F_{g}=F_{g} \quad \text { as } z \widehat{\rightarrow} t_{0},
$$


which, together with (5.24), gives (5.21). Upon applying the standard estimates

$$
\left\|f_{ \pm}(z)\right\|_{\mathcal{Y}} \leq \frac{\|f\|_{H^{w}}}{\sqrt{1-|z|^{2}}} \quad\left(f=\left[\begin{array}{c}
f_{+} \\
f_{-}
\end{array}\right] \in H^{w}, \quad z \in \mathbb{D}\right)
$$

to the function $f=F_{g}-L_{z}^{(n)} g$, we get

$$
\left\|F_{g, \pm}(\zeta)-L_{z, \pm}^{(n)}(\zeta) g\right\|_{\mathcal{Y}} \leq \frac{\left\|F_{g}-L_{z}^{(n)} g\right\|_{H^{w}}}{\sqrt{1-|\zeta|^{2}}} .
$$

This, together with (5.21), implies that

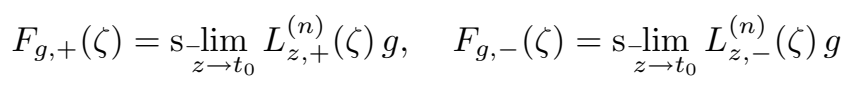

for every $\zeta \in \mathbb{D}$; thus,

$$
F_{g}(\zeta)=\mathrm{s}_{z \rightarrow t_{0}} L_{z}^{(n)}(\zeta) g
$$

Now we repeat the arguments used to derive (5.19) from (5.15). However, since now we start with the strong limit (5.27) rather than the weak subsequential limit (5.15), the conclusion strengthening (5.19) is that for every $g \in \mathcal{G}$, the strong limits $u_{j}=\mathrm{s}_{z \rightarrow t_{0}} \mathbf{b}_{j}(z)^{*} g$ exist for $j=0, \ldots, n$.

Define operators $\mathbf{b}_{j}\left(t_{0}\right)^{*}: \mathcal{G} \rightarrow \mathcal{U}$ by

$$
\mathbf{b}_{j}\left(t_{0}\right)^{*} g=\mathrm{s}-l i m_{z \rightarrow t_{0}} \mathbf{b}_{j}(z)^{*} g \quad(j=0, \ldots, n) .
$$

It is clear that $\mathbf{b}_{j}\left(t_{0}\right)^{*}$ is a linear operator defined on all of $\mathcal{G}$. Thus, by the BanachSteinhaus theorem, it is bounded. This proves statement (1) in the theorem. Again, let $g \in \mathcal{G}$ be fixed. Substituting $u_{j}=\mathbf{b}_{j}\left(t_{0}\right)^{*} g$ into (5.20) and comparing the resulting formula with (5.9), we see that $L_{t_{0}}^{(n)} g=F_{g}$. Therefore, $L_{t_{0}}^{(n)} g$ belongs to $H^{w}$ for every $g \in \mathcal{G}$. It now follows from (5.21) that $L_{z}^{(n)} g \stackrel{H^{w}}{\longrightarrow} L_{t_{0}}^{(n)} g$, which completes the proof of statements (1) and of statement (2) for $j=n$. To complete the proof of statement (2), we take advantage of the recursive relation

$$
L_{t_{0}}^{(j-1)}(t)=\frac{1}{t}\left(\left(1-t \bar{t}_{0}\right) L_{t_{0}}^{(j)}(t)-\left[\begin{array}{cc}
\mathrm{I}_{\mathcal{Y}} & w(t) \\
w(t)^{*} & \mathrm{I}_{\mathcal{U}}
\end{array}\right]\left[\begin{array}{c}
\mathbf{a}_{j}\left(t_{0}\right)^{*} \\
-\mathbf{b}_{j}\left(t_{0}\right)^{*}
\end{array}\right]\right),
$$

verification of which is straightforward, and show that

$$
L_{t_{0}}^{(j)} g \in H^{w} \quad \Longrightarrow \quad L_{t_{0}}^{(j-1)} g \in H^{w} .
$$

Indeed, if $L_{t_{0}}^{(j)} g \in H^{w}$, then in particular, $L_{t_{0}}^{(j)} g \in L^{w}$; and it follows from (5.28) that $L_{t_{0}}^{(j-1)} g \in L^{w}$. Furthermore, by (5.28),

$$
L_{t_{0},+}^{(j-1)}(\zeta) g=f_{+}(\zeta) / \zeta
$$


where

$$
f_{+}(\zeta):=\left(1-\zeta \bar{t}_{0}\right) L_{t_{0},+}^{(j)}(\zeta) g-\mathbf{a}_{j}\left(t_{0}\right)^{*} g+w(\zeta) \mathbf{b}_{j}\left(t_{0}\right)^{*} g
$$

The function $f_{+}$belongs to $H_{2}^{+}(\mathcal{Y})$ since $L_{t_{0},+}^{(j)} g \in H_{2}^{+}(\mathcal{Y})$. Since

$$
f_{+}(0)=L_{t_{0},+}^{(j)}(0) g-\mathbf{a}_{j}\left(t_{0}\right)^{*} g+w(0) \mathbf{b}_{j}\left(t_{0}\right)^{*} g=0
$$

the function $L_{t_{0},+}^{(j-1)} g=f_{+}(\zeta) / \zeta$ also belongs to $H_{2}^{+}(\mathcal{Y})$. Comparing the bottom components in (5.28), we get

$$
L_{t_{0},-}^{(j-1)}(\zeta) g=\left(\bar{\zeta}-\bar{t}_{0}\right) L_{t_{0},-}^{(j)}(\zeta) g-\bar{\zeta} w(\zeta)^{*} \mathbf{a}_{j}\left(t_{0}\right)^{*} g+\bar{\zeta} \mathbf{b}_{j}\left(t_{0}\right)^{*} g
$$

thus, the assumption $L_{t_{0},-}^{(j)} g \in H_{2}^{-}(\mathcal{U})$ implies that $L_{t_{0},-}^{(j-1)} g \in H_{2}^{-}(\mathcal{U})$. Therefore, $L_{t_{0}}^{(j-1)} g \in H^{w}$, by Definition 4.1. Since the membership $L_{t_{0}}^{(n)} g \in H^{w}$ is already proved, the inverse induction arguments show that $L_{t_{0}}^{(j)} g \in H^{w}$ for every $g \in \mathcal{G}$ and $j \in\{0, \ldots, n\}$. Repeating the arguments used to get (5.21) (now with $j$ replacing $n$ ), we conclude that (5.12) holds for every $j=0, \ldots, n$.

To prove statement (3), we note that by formula (5.8) and in view of (5.12),

$$
\text { (5.30) } \lim _{z \widehat{\rightarrow} t_{0}}\left\langle\mathbf{P}_{n n}^{w, \mathbf{a}}(z) g, g\right\rangle_{\mathcal{G}}=\lim _{z \widehat{\rightarrow} t_{0}}\left\|L_{z}^{(n)} g\right\|_{H^{w}}^{2}=\left\|\lim _{z \widehat{\rightarrow} t_{0}} L_{z}^{(n)} g\right\|_{H^{w}}^{2}=\left\|L_{t_{0}}^{(n)} g\right\|_{H^{w}}^{2},
$$

which proves the existence of the weak limit in (5.13). Observe that the inequality

$$
\liminf _{z \rightarrow t_{0}}\left\langle\mathbf{P}_{n n}^{w, \mathbf{a}}(z) g, g\right\rangle \leq \lim _{z \breve{\rightarrow} t_{0}}\left\langle\mathbf{P}_{n n}^{w, \mathbf{a}}(z) g, g\right\rangle
$$

is obvious, since the first limit allows $z$ to approach $t_{0}$ unrestrictedly in $\mathbb{D}$ (more precisely, unrestrictedly in the neighborhood $\mathcal{U}_{t_{0}, \varepsilon}$ of $t_{0}$ of the form (2.1) on which a is analytic), while the second limit is nontangential. To prove the reverse inequality, assume that $\left\{z_{j}\right\}$ is a sequence that leads to the limit inferior in (5.14). Then the sequence of numbers $\left\|L_{z_{j}}^{(n)} g\right\|_{H^{w}}^{2}$ converges to the limit inferior and, in particular, is bounded. Thus there exists a subsequence of the sequence $\left\{z_{j}\right\}$ (still denoted by $\left\{z_{j}\right\}$ ) such that $L_{z_{j}}^{(n)} g$ converges to $L_{t_{0}}^{(n)} g$ weakly in $H^{w}$. Then

$$
\left\|L_{t_{0}}^{(n)} g\right\|_{H^{w}}^{2} \leq \lim _{z_{j} \rightarrow t_{0}}\left\|L_{z_{j}}^{(n)} g\right\|_{H^{w}}^{2}=\liminf _{z \rightarrow t_{0}}\left\langle\mathbf{P}_{n n}^{w, \mathbf{a}}(z) g, g\right\rangle
$$

Combining the latter inequality with (5.30) gives

$$
\lim _{z \rightarrow t_{0}}\left\langle\mathbf{P}_{n n}^{w, \mathbf{a}}(z) g, g\right\rangle_{\mathcal{G}}=\left\|L_{t_{0}}^{(n)} g\right\|_{H^{w}}^{2} \leq \liminf _{z \rightarrow t_{0}}\left\langle\mathbf{P}_{n n}^{w, \mathbf{a}}(z) g, g\right\rangle
$$

which, together with (5.31), implies (5.14) and completes the proof. 
In the next section, we make extensive use of the polynomials

$$
A_{j}(z)=\sum_{\ell=0}^{j} z^{j-\ell}\left(1-z \bar{t}_{0}\right)^{\ell} \mathbf{a}_{\ell}\left(t_{0}\right)^{*}, \quad B_{j}(z)=\sum_{\ell=0}^{j} z^{j-\ell}\left(1-z \bar{t}_{0}\right)^{\ell} \mathbf{b}_{\ell}\left(t_{0}\right)^{*},
$$

which can be written in terms of the formula (2.12) as $p_{j}^{\mathbf{a} \sharp}$ and $p_{j}^{\mathbf{b} \sharp}$, respectively. Here we just note that formula (5.9) for $L_{t_{0}}^{(j)}$ can be written in terms of these polynomials as

$$
L_{t_{0}}^{(j)}(t)=\left[\begin{array}{cc}
\mathrm{I}_{\mathcal{Y}} & w(t) \\
w(t)^{*} & \mathrm{I}_{\mathcal{U}}
\end{array}\right]\left[\begin{array}{r}
A_{j}(t) \\
-B_{j}(t)
\end{array}\right] \frac{1}{\left(1-t \bar{t}_{0}\right)^{j+1}} .
$$

\section{The kernels $\widetilde{L}_{z}^{(j)}$ and their boundary analogues}

The kernels $L_{z}^{(j)}$ were introduced in Section 4 as certain tangential analogues of the kernels $K_{z}^{(j)}$ defined in (4.7). In this section, we introduce the kernels $\widetilde{L}_{z}^{(j)}$, which are tangential counterparts of the kernels $\widetilde{K}_{z}^{(j)}$ also defined in (4.7). The construction is carried out under the assumption (3.5), so that we have at our disposal the operators $\mathbf{a}_{\ell}\left(t_{0}\right)=\mathbf{a}^{(\ell)}\left(t_{0}\right) / \ell ! \in \mathcal{L}(\mathcal{Y}, \mathcal{G})$ for all $\ell \in \mathbb{Z}_{+}$and $\mathbf{b}_{\ell}\left(t_{0}\right)^{*}=\mathrm{s}_{z \widehat{\rightarrow} t_{0}}[\mathbf{a}(z) w(z)]_{j}^{*} \in \mathcal{L}(\mathcal{G}, \mathcal{U})$ for $\ell=0, \ldots, n$. Therefore, we can introduce the polynomials (5.32) for $j=0, \ldots, n$ and their derivatives

$$
A_{j, i}(z):=\frac{1}{i !} \frac{d^{i}}{d z^{i}} A_{j}(z) \quad \text { and } \quad B_{j, i}(z):=\frac{1}{i !} \frac{d^{i}}{d z^{i}} B_{j}(z)
$$

so that

$$
A_{j}(z)=\sum_{i=0}^{j} A_{j, i}\left(t_{0}\right)\left(z-t_{0}\right)^{i} \quad \text { and } \quad B_{j}(z)=\sum_{i=0}^{j} B_{j, i}\left(t_{0}\right)\left(z-t_{0}\right)^{i} .
$$

Now we introduce the kernels

$$
\widetilde{L}_{z}^{(j)}(t):=\frac{1}{j !} \frac{\partial^{j}}{\partial z^{j}}\left(\widetilde{K}_{z}(t) B_{j}(z)\right)=\sum_{i=0}^{j} \widetilde{K}_{z}^{(i)}(t) B_{j, j-i}(z)
$$

for $j=0, \ldots, n$, where $\widetilde{K}_{z}$ is given by (4.6) and where the second equality follows by the Leibnitz rule. Making use of (4.6) and of the functions

$$
\mathbf{c}_{j}(z)=w(z) B_{j}(z) \quad(j=0, \ldots, n),
$$

we can write

$$
\widetilde{K}_{z}(t) B_{j}(z)=\left[\begin{array}{cc}
\mathrm{I}_{\mathcal{Y}} & w(t) \\
w(t)^{*} & \mathrm{I}_{\mathcal{U}}
\end{array}\right]\left[\begin{array}{r}
-\mathbf{c}_{j}(z) \\
B_{j}(z)
\end{array}\right] \cdot \widetilde{k}_{z}(t)
$$


another application of Leibnitz rule leads us to

$$
\widetilde{L}_{z}^{(j)}(t)=\left[\begin{array}{cc}
\mathrm{I}_{\mathcal{Y}} & w(t) \\
w(t)^{*} & \mathrm{I}_{\mathcal{U}}
\end{array}\right] \sum_{i=0}^{j}\left[\begin{array}{c}
-\mathbf{c}_{j, j-i}(z) \\
B_{j, j-i}(z)
\end{array}\right] \widetilde{k}_{i, z}(t)
$$

The next lemma establishes a reproducing property of the kernel $\widetilde{L}_{z}^{(j)}(t)$.

Lemma 6.1. For every $j \in \mathbb{Z}_{+}, g \in \mathcal{G}$ and $z \in \mathbb{D}$, the function $\widetilde{L}_{z}^{(i)} \mathrm{g}$ belongs to $H^{w}$; furthermore, for every $f=\left[\begin{array}{l}f_{+} \\ f_{-}\end{array}\right] \in H^{w}$,

$$
\left\langle f, \widetilde{L}_{z}^{(j)} g\right\rangle_{H^{w}}=\frac{1}{j !}\left\langle\frac{d^{j}}{d \bar{z}^{j}}\left(\frac{B_{j}(z)^{*} f_{-}(z)}{\bar{z}}\right), g\right\rangle_{\mathcal{G}} .
$$

Proof. By Lemma 4.2, $\widetilde{K}_{z}^{(i)} u_{i}$ belongs to the space $H^{w}$ for every $u_{i} \in \mathcal{U}$. Upon choosing $u_{i}=B_{j, j-i}(z) g$, we get the first statement directly from (6.3). Using the reproducing property (4.9), we get (6.6):

$$
\begin{aligned}
\left\langle f, \widetilde{L}_{z}^{(j)} g\right\rangle_{H^{w}} & =\frac{1}{j !} \frac{d^{j}}{d \bar{z}^{j}}\left\langle f, \widetilde{K}_{z} B_{j}(z) g\right\rangle_{H^{w}} \\
& =\frac{1}{j !} \frac{d^{j}}{d \bar{z}^{j}}\left\langle\frac{f_{-}(z)}{\bar{z}}, B_{j}(z) g\right\rangle_{\mathcal{U}}=\frac{1}{j !}\left\langle\frac{d^{j}}{d \bar{z}^{j}}\left(\frac{B_{j}(z)^{*} f_{-}(z)}{\bar{z}}\right), g\right\rangle_{\mathcal{G}}
\end{aligned}
$$

To extend definitions (6.5) of $\widetilde{L}_{z}^{(j)}$ to the boundary by continuity (that is, letting $z \widehat{\rightarrow} t_{0}$ in (6.5)), we need to be sure that the functions

$$
\mathbf{c}_{j, i}(z)=\frac{1}{i !} \frac{d^{i}}{d z^{i}} \mathbf{c}_{j}(z)=\frac{1}{i !} \frac{d^{i}}{d z^{i}}\left(w(z) B_{j}(z)\right)
$$

possess at least weak angular limits at $t_{0}$. The next theorem shows that condition (3.5) guarantees the existence of such limits even in the strong sense.

Theorem 6.2. Assume that condition (3.5) holds for $w \in \mathcal{S}(\mathcal{U}, \mathcal{Y})$ and an $\mathcal{L}(\mathcal{Y}, \mathcal{G})$ valued function a analytic at $t_{0} \in \mathbb{T}$. Let $A_{j}$ and $B_{j}$ be the polynomials defined in (5.32). Then the strong nontangential boundary limits

$$
\mathbf{c}_{j, i}\left(t_{0}\right):=\frac{1}{i !} \cdot \mathrm{s}-\lim _{z \rightarrow t_{0}}\left(w(z) B_{j}(z)\right)^{(i)} \quad(0 \leq i \leq j \leq n)
$$

exist; moreover,

$$
\mathbf{c}_{j, i}\left(t_{0}\right)=A_{j, i}\left(t_{0}\right) \quad \text { for } 0 \leq i \leq j \leq n
$$


Proof. Fix $j \in\{0, \ldots, n\}$ and $g \in \mathcal{G}$. By Theorem 5.3, $L_{t_{0}}^{(j)} g$ belongs to $H^{w}$; therefore, its top component $L_{t_{0},+}^{(j)} g$ belongs to $H_{2}^{+}(\mathcal{Y})$. By formula (5.33),

$$
L_{t_{0},+}^{(j)}(t) g=\frac{A_{j}(t)-w(t) B_{j}(t)}{\left(1-t \bar{t}_{0}\right)^{j+1}} g .
$$

Now we apply the standard $H_{2}$ estimate

$$
\left\|L_{t_{0},+}^{(j)}(\zeta) g\right\|_{\mathcal{Y}} \leq\left(1-|\zeta|^{2}\right)^{-1 / 2} \cdot\left\|L_{t_{0},+}^{(j)} g\right\|_{H_{2}^{+}(\mathcal{Y})}
$$

to the analytic extension of $L_{t_{0},+}^{(j)} g$ inside the unit disk to get

$$
\left\|\left(A_{j}(\zeta)-w(\zeta) B_{j}(\zeta)\right) g\right\| \leq \frac{\left|1-\zeta \bar{t}_{0}\right|^{j+1}}{\sqrt{1-|\zeta|^{2}}} \cdot\left\|L_{t_{0},+}^{(j)} g\right\|_{H_{2}^{+}(\mathcal{Y})} .
$$

Therefore,

$$
\left\|\left(A_{j}(\zeta)-w(\zeta) B_{j}(\zeta)\right) g\right\|=o\left(\left|\zeta-t_{0}\right|^{j}\right) \quad \text { as } z \widehat{\rightarrow} t_{0} .
$$

Consequently,

$$
\mathrm{s}-\lim _{z \rightarrow t_{0}} \frac{d^{i}}{d \zeta^{i}}\left(A_{j}(\zeta)-w(\zeta) B_{j}(\zeta)\right) g=0 \quad \text { for } i=0, \ldots, j .
$$

Since $g \in \mathcal{G}$ is arbitrary, since $A_{j}$ is a polynomial and since by (6.2)

$$
\frac{1}{i !} \cdot \lim _{z \rightarrow t_{0}} \frac{d^{i}}{d \zeta^{i}} A_{j}(\zeta)=A_{j, i}\left(t_{0}\right)
$$

(6.9) implies (6.7) and (6.8).

Now we introduce the boundary kernels $\widetilde{L}_{t_{0}}^{(j)}$ upon letting $z \rightarrow t_{0}$ in (6.5):

$$
\widetilde{L}_{t_{0}}^{(j)}(t)=\left[\begin{array}{cc}
\mathrm{I}_{\mathcal{Y}} & w(t) \\
w(t)^{*} & \mathrm{I}_{\mathcal{U}}
\end{array}\right] \sum_{i=0}^{j}\left[\begin{array}{r}
-A_{j, j-i}\left(t_{0}\right) \\
B_{j, j-i}\left(t_{0}\right)
\end{array}\right] \widetilde{k}_{i, t_{0}}(t) .
$$

Since, by (6.2),

$$
\begin{aligned}
\sum_{i=0}^{j}\left[\begin{array}{r}
-A_{j, j-i}\left(t_{0}\right) \\
B_{j, j-i}\left(t_{0}\right)
\end{array}\right] \widetilde{k}_{i, t_{0}}(t) & =\widetilde{k}_{j, t_{0}}(t) \cdot \sum_{i=0}^{j}\left[\begin{array}{r}
-A_{j, i}\left(t_{0}\right) \\
B_{j, i}\left(t_{0}\right)
\end{array}\right]\left(t-t_{0}\right)^{i} \\
& =\left[\begin{array}{r}
-A_{j}(t) \\
B_{j}(t)
\end{array}\right] \widetilde{k}_{j, t_{0}}(t),
\end{aligned}
$$

we can rewrite (6.10) as

$$
\widetilde{L}_{t_{0}}^{(j)}(t)=\left[\begin{array}{cc}
\mathrm{I}_{\mathcal{Y}} & w(t) \\
w(t)^{*} & \mathrm{I}_{\mathcal{U}}
\end{array}\right]\left[\begin{array}{r}
-A_{j}(t) \\
B_{j}(t)
\end{array}\right] \widetilde{k}_{j, t_{0}}(t) .
$$

Comparing the latter formula with (5.33) leads to the conclusion that

$$
L_{t_{0}}^{(j)}(t)=(-1)^{j} t_{0}^{j+1} \widetilde{L}_{t_{0}}^{(j)}(t) \quad \text { for } j=0, \ldots, n .
$$


Theorem 6.3. Assume that (3.5) holds for $w \in \mathcal{S}(\mathcal{U}, \mathcal{Y})$ and an $\mathcal{L}(\mathcal{Y}, \mathcal{G})$-valued function a analytic at $t_{0}$, and let $\widetilde{L}_{z}^{(j)}$ and $\widetilde{L}_{t_{0}}^{(j)}$ be the kernels introduced in (6.5) and (6.10), respectively. Then for every vector $g \in \mathcal{G}$, the function $\widetilde{L}_{t_{0}}^{(j)}(t) \mathrm{g}$ belongs to $H^{w}$, and $\widetilde{L}_{z}^{(j)} g \stackrel{H^{w}}{\longrightarrow} \widetilde{L}_{t_{0}}^{(j)} g$ as $z \widehat{\rightarrow} t_{0}$.

Proof. If condition (3.5) holds, then $L_{t_{0}}^{(j)} g$ belongs to $H^{w}$ by Theorem 5.3. Then it follows by (6.12), that

$$
h(t):=\widetilde{L}_{t_{0}}^{(j)}(t) g
$$

belongs to $H^{w}$ as well. Furthermore, by Lemma 4.5,

$$
\widetilde{h}_{z}(t)=\left(\frac{t-t_{0}}{t-z}\right)^{j+1} h(t)=\left(\frac{t-t_{0}}{t-z}\right)^{j+1} \widetilde{L}_{t_{0}}^{(j)}(t) g
$$

belongs to $L^{w}$ and tends to $h$ in norm of $L^{w}$ as $z \widehat{\rightarrow} t_{0}$. Then $P_{H^{w}} \widetilde{h}_{z}$ (the orthogonal projection of $\widetilde{h}_{z}$ onto $H^{w}$ ) belongs to $H^{w}$ and tends to $h$ in norm of $H^{w}$ as $z \widehat{\rightarrow} t_{0}$. Thus, to complete the proof, it suffices to show that

$$
P_{H w} \widetilde{h}_{z}=\widetilde{L}_{z}^{(j)} g .
$$

To this end, we use the formula (6.11) for $\widetilde{L}_{t_{0}}^{(j)}$ to rewrite (6.13) in the form

$$
\widetilde{h}_{z}(t)=\left[\begin{array}{cc}
\mathrm{I}_{\mathcal{Y}} & w(t) \\
w(t)^{*} & \mathrm{I}_{\mathcal{U}}
\end{array}\right]\left[\begin{array}{r}
-A_{j}(t) \\
B_{j}(t)
\end{array}\right] \frac{1}{(t-z)^{j+1}} .
$$

By construction (6.2), $A_{j}$ is a polynomial in $t \in \mathbb{T}$ of degree $j$; thus the function

$$
\frac{A_{j}(t) g}{(t-z)^{j+1}}=\frac{\bar{t}^{j+1} A_{j}(t) g}{(1-\bar{t} z)^{j+1}} \quad \text { belongs to } H_{2}^{-}(\mathcal{Y}) \text {. }
$$

Then by formula (4.13) in Lemma 4.4,

$$
P_{H^{w}} \widetilde{h}_{z}=P_{H^{w}}\left[\begin{array}{cc}
\mathrm{I}_{\mathcal{Y}} & w(t) \\
w(t)^{*} & \mathrm{I}_{\mathcal{U}}
\end{array}\right]\left[\begin{array}{c}
0 \\
B_{j}(t) g
\end{array}\right] \frac{1}{(t-z)^{j+1}} .
$$

Note that by (6.2),

$$
\begin{aligned}
\frac{B_{j}(t)}{(t-z)^{j+1}} & =\sum_{i=0}^{j} B_{j, j-i}\left(t_{0}\right)\left(t-t_{0}\right)^{j-i} \frac{1}{(t-z)^{j+1}} \\
& =\sum_{i=0}^{j} \sum_{\ell=0}^{j-i}\left(\begin{array}{c}
j-i \\
\ell
\end{array}\right) B_{j, j-i}\left(t_{0}\right) \frac{\left(z-t_{0}\right)^{\ell}}{(t-z)^{i+\ell+1}} \\
& =\sum_{k=0}^{j} \frac{1}{(t-z)^{k+1}} \sum_{i+\ell=k}\left(\begin{array}{c}
j-i \\
\ell
\end{array}\right) B_{j, j-i}\left(t_{0}\right)\left(z-t_{0}\right)^{\ell} .
\end{aligned}
$$


Substituting the latter relation into (6.15) and then making use of (4.11), we get

$$
\begin{aligned}
P_{H^{w}} \widetilde{h}_{z}= & \sum_{k=0}^{j} P_{H^{w}}\left(\left[\begin{array}{cc}
\mathrm{I}_{\mathcal{Y}} & w(t) \\
w(t)^{*} & \mathrm{I}_{\mathcal{U}}
\end{array}\right]\left[\begin{array}{c}
0 \\
\frac{I_{\mathcal{U}}}{(t-z)^{k+1}}
\end{array}\right]\right) \\
& \times \sum_{i+\ell=k}\left(\begin{array}{c}
j-i \\
\ell
\end{array}\right) B_{j, j-i}\left(t_{0}\right)\left(z-t_{0}\right)^{\ell} \\
= & \sum_{k=0}^{j} \widetilde{K}_{z}^{(k)}(t) \sum_{i+\ell=k}\left(\begin{array}{c}
j-i \\
\ell
\end{array}\right) B_{j, j-i}\left(t_{0}\right)\left(z-t_{0}\right)^{\ell} g .
\end{aligned}
$$

On the other hand, differentiating the second equality in (6.2), we get

$$
B_{j, i}(z)=\frac{1}{i !} \frac{d^{i}}{d z^{i}} B_{j}(z)=\sum_{\ell=0}^{j-i}\left(\begin{array}{c}
i+\ell \\
\ell
\end{array}\right) B_{j, i+\ell}\left(t_{0}\right)\left(z-t_{0}\right)^{\ell}
$$

therefore,

$$
B_{j, j-k}(z)=\sum_{i+\ell=k}\left(\begin{array}{c}
j-\ell \\
\ell
\end{array}\right) B_{j, j-i}\left(t_{0}\right)\left(z-t_{0}\right)^{\ell} .
$$

Substituting the latter formula into (6.16), we obtain

$$
P_{H^{w}} \widetilde{h}_{z}=\sum_{k=0}^{j} \widetilde{K}_{z}^{(k)}(t) B_{j, j-k}(t) g
$$

which, together with (6.3), implies (6.14). This completes the proof of the theorem.

\section{Proof of Theorem 3.1}

Now we are in a position to give a proof of Theorem 3.1. Since statements (2) and (3) have been already proved in Theorems 5.3 and 6.2, respectively, it remains to prove the two other statements. First we recall that, by Theorems 5.3 and 6.3 , the functions $L_{t_{0}}^{(j)} g$ and $\widetilde{L}_{t_{0}}^{(j)} g$ defined via formulas (5.9) and (6.10) belong to $H^{w}$ for every $j \in\{0, \ldots, n\}, g \in \mathcal{G}$ and, furthermore,

$$
L_{z}^{(j)} g \stackrel{H^{w}}{\longrightarrow} L_{t_{0}}^{(j)} g \quad \text { and } \quad \widetilde{L}_{z}^{(j)} g \stackrel{H^{w}}{\longrightarrow} \widetilde{L}_{t_{0}}^{(j)} g \quad \text { as } z \widehat{\rightarrow} t_{0}
$$

Writing

$$
\begin{aligned}
\mathbf{L}_{z} & =\left[\begin{array}{lll}
L_{z}^{(0)} & \ldots & L_{z}^{(n)}
\end{array}\right], & \widetilde{\mathbf{L}}_{z} & =\left[\begin{array}{llll}
\widetilde{L}_{z}^{(0)} & \ldots & \widetilde{L}_{z}^{(n)}
\end{array}\right], \\
\mathbf{L}_{t_{0}} & =\left[\begin{array}{lllll}
L_{t_{0}}^{(0)} & \ldots & L_{t_{0}}^{(n)}
\end{array}\right], & \widetilde{\mathbf{L}}_{t_{0}} & =\left[\begin{array}{llll}
\widetilde{L}_{t_{0}}^{(0)} & \ldots & \widetilde{L}_{t_{0}}^{(n)}
\end{array}\right],
\end{aligned}
$$


we can express (7.1) equivalently as

$$
\mathbf{L}_{z} \mathbf{g} \stackrel{H^{w}}{\longrightarrow} \mathbf{L}_{t_{0}} \mathbf{g} \quad \text { and } \quad \widetilde{\mathbf{L}}_{z} \mathbf{g} \stackrel{H^{w}}{\longrightarrow} \widetilde{\mathbf{L}}_{t_{0}} \mathbf{g} \quad\left(z \widehat{\rightarrow} t_{0}\right),
$$

holding for every vector $\mathrm{g} \in \mathcal{G}^{n+1}$.

Proof of statement (1). We first note that (5.8) for the block entries $\mathbf{P}_{i, j}^{w, \mathbf{a}}(z)$ of the tangential Schwarz-Pick matrix $\mathbf{P}_{n}^{w, \mathbf{a}}(z)$ can be equivalently expressed as a single equality

$$
\left\langle\mathbf{L}_{z} \mathbf{g}^{\prime}\right\rangle_{H^{w}}=\left\langle\mathbf{P}_{n}^{w, \mathbf{a}}(z) \mathbf{g}, \mathbf{g}^{\prime}\right\rangle_{\mathcal{G}^{n+1}}
$$

holding for every $\mathbf{g}, \mathbf{g}^{\prime}$, where $\mathbf{L}_{z}$ is given in (7.2). By the first convergence in (7.4), we have

$$
\lim _{z \rightarrow t_{0}}\left\langle\mathbf{L}_{z} \mathbf{g}, \mathbf{L}_{z} \mathbf{g}^{\prime}\right\rangle_{H^{w}}=\left\langle\lim _{z \rightarrow t_{0}} \mathbf{L}_{z} \mathbf{g}, \lim _{z \rightarrow t_{0}} \mathbf{L}_{z} \mathbf{g}^{\prime}\right\rangle_{H^{w}}=\left\langle\mathbf{L}_{t_{0}} \mathbf{g}, \mathbf{L}_{t_{0}} \mathbf{g}^{\prime}\right\rangle_{H^{w}},
$$

which together with (7.5) implies (since $g$ and $\mathbf{g}^{\prime}$ are arbitrary) that the weak limit $\mathbf{P}_{n}^{w, \mathbf{a}}\left(t_{0}\right):=\underset{z}{ }-\lim _{z \rightarrow t_{0}} \mathbf{P}_{n}^{w, \mathbf{a}}(z)$ exists and is uniquely defined by the equality

$$
\left\langle\mathbf{P}_{n}^{w, \mathbf{a}}\left(t_{0}\right) \mathbf{g}, \mathbf{g}^{\prime}\right\rangle_{\mathcal{G}^{n+1}}=\left\langle\mathbf{L}_{t_{0}} \mathbf{g}, \mathbf{L}_{t_{0}} \mathbf{g}^{\prime}\right\rangle_{H^{w}}, \quad \mathbf{g}, \mathbf{g}^{\prime} \in \mathcal{G}^{n+1} .
$$

Proof of statement (4). From definitions (2.8), (3.4) and (2.14) of $\mathbf{H}_{n}(z)$, $\mathbf{T}_{n}^{\mathbf{a}}(z)$ and $\mathbf{U}_{n}^{\mathbf{b}}(z)$ respectively, it follows that the $i j$-th block entry $\mathbf{R}_{i j}(z)$ of the matrix $\mathbf{R}(z):=\mathbf{T}_{n}^{\mathbf{a}}(z) \mathbf{H}_{n}(z) \mathbf{U}_{n}^{\mathrm{b}}(z)$ equals

$$
\mathbf{R}_{i j}(z)=\sum_{\ell=0}^{j} \sum_{k=0}^{i} \mathbf{a}_{j-\ell}(z) w_{\ell+k+1}(z) B_{i, i-k}(z) \quad(i, j=0, \ldots, n) .
$$

On the other hand, it follows from (5.2) and (6.3), that for every $g, g^{\prime} \in \mathcal{G}$,

$$
\begin{aligned}
\left\langle\widetilde{L}_{z}^{(i)} g, L_{z}^{(j)} g^{\prime}\right\rangle_{H^{w}} & =\left\langle\sum_{k=0}^{i} \widetilde{K}_{z}^{(k)}(t) B_{i, i-k}(z) g, \sum_{\ell=0}^{j} K_{z}^{(\ell)}(t) \mathbf{a}_{j-\ell}(z)^{*} g^{\prime}\right\rangle_{H^{w}} \\
& =\sum_{\ell=0}^{j} \sum_{k=0}^{i}\left\langle\widetilde{K}_{z}^{(k)}(t) B_{i, i-k}(z) g, K_{z}^{(\ell)}(t) \mathbf{a}_{j-\ell}(z)^{*} g^{\prime}\right\rangle_{H^{w}} .
\end{aligned}
$$

Recall the equality

$$
\left\langle\widetilde{K}_{z}^{(k)} u, K_{z}^{(\ell)} y\right\rangle_{H^{w}}=\left\langle w_{k+\ell+1}(z) u, y\right\rangle_{\mathcal{Y}}
$$

holding for every $z \in \mathbb{D}, k, \ell \in \mathbb{Z}_{+}, u \in \mathcal{U}$, and $y \in \mathcal{Y}$ (see Lemma 2.3 in [4] for the proof). Letting $u=B_{i, i-k}(z) g$ and $y=\mathbf{a}_{j-\ell}(z)^{*} g^{\prime}$ in this equality, we get

$$
\begin{aligned}
\left\langle\widetilde{K}_{z}^{(k)} B_{i, i-k}(z), K_{z}^{(i)} \mathbf{a}_{j-\ell}(z)^{*} g^{\prime}\right\rangle_{H^{w}} & =\left\langle w_{k+\ell+1}(z) B_{i, i-k}(z) g^{\prime}, \mathbf{a}_{j-\ell}(z)^{*} g\right\rangle_{\mathcal{G}} \\
& =\left\langle\mathbf{a}_{j-\ell}(z) w_{k+\ell+1}(z) B_{i, i-k}(z) g, g^{\prime}\right\rangle_{\mathcal{G}} .
\end{aligned}
$$


Substituting the latter equalities into the right hand side of (7.8) and making use of (7.7) leads to

$$
\left\langle\widetilde{L}_{z}^{(i)} g^{\prime}, L_{z}^{(j)} g\right\rangle_{H^{w}}=\left\langle\mathbf{R}_{i j}(z) g, g^{\prime}\right\rangle_{\mathcal{G}} \quad\left(i, j=0, \ldots, n ; g, g^{\prime} \in \mathcal{G}\right)
$$

which can be written equivalently in terms of (7.2) as

$$
\left\langle\widetilde{\mathbf{L}}_{z} \mathbf{g}, \mathbf{L}_{z} \mathbf{g}^{\prime}\right\rangle_{H^{w}}=\left\langle\mathbf{R}(z) \mathbf{g}, \mathbf{g}^{\prime}\right\rangle_{\mathcal{G}^{n+1}}=\left\langle\mathbf{T}_{n}^{\mathbf{a}}(z) \mathbf{H}_{n}(z) \mathbf{U}_{n}^{\mathbf{b}}(z) \mathbf{g}, \mathbf{g}^{\prime}\right\rangle_{\mathcal{G}^{n+1}},
$$

holding for all vectors $\mathbf{g}, \mathbf{g}^{\prime} \in \mathcal{G}^{n+1}$. Since matrix $\mathbf{M}$ given by (2.17) is unitary, we can replace $\mathrm{g}$ by $\mathrm{Mg}$ to get

$$
\left\langle\widetilde{\mathbf{L}}_{z} \mathbf{M g}, \mathbf{L}_{z} \mathbf{g}^{\prime}\right\rangle_{H^{w}}=\left\langle\mathbf{T}_{n}^{\mathbf{a}}(z) \mathbf{H}_{n}(z) \mathbf{U}_{n}^{\mathbf{b}}(z) \mathbf{M g}, \mathbf{g}^{\prime}\right\rangle_{\mathcal{G}^{n+1}},
$$

also holding for all $\mathbf{g}, \mathrm{g}^{\prime} \in \mathcal{G}^{n+1}$. By (7.4), we can pass in (7.9) to the limits as $z \widehat{\rightarrow} t_{0}$ to conclude (as in the proof of statement (1) above) that the weak limit $\mathbb{P}_{n}^{w, \mathbf{a}}\left(t_{0}\right)$ in (3.8) exists and satisfies

$$
\left\langle\mathbb{P}_{n}^{w, \mathbf{a}}\left(t_{0}\right) \mathbf{g}, \mathbf{g}^{\prime}\right\rangle_{\mathcal{G}^{n+1}}=\left\langle\widetilde{\mathbf{L}}_{t_{0}} \mathbf{M g}, \mathbf{L}_{t_{0}} \mathbf{g}^{\prime}\right\rangle_{H^{w}} \quad \text { for all } \mathbf{g}, \mathbf{g}^{\prime} \in \mathcal{G}^{n+1}
$$

Note that relations (6.13) can be written in matrix form as

$$
\mathbf{L}_{t_{0}}=\widetilde{\mathbf{L}}_{t_{0}} \mathbf{M}
$$

using which we combine (7.10) and (7.6) to get

$$
\left\langle\mathbb{P}_{n}^{w, \mathbf{a}}\left(t_{0}\right) \mathbf{g}, \mathbf{g}^{\prime}\right\rangle=\left\langle\widetilde{\mathbf{L}}_{t_{0}} \mathbf{M g}, \mathbf{L}_{t_{0}} \mathbf{g}^{\prime}\right\rangle_{H^{w}}=\left\langle\mathbf{L}_{t_{0}} \mathbf{g}, \mathbf{L}_{t_{0}} \mathbf{g}^{\prime}\right\rangle_{H^{w}}=\left\langle\mathbf{P}_{n}^{w, \mathbf{a}}\left(t_{0}\right) \mathbf{g}, \mathbf{g}^{\prime}\right\rangle .
$$

Since the latter holds for every $\mathbf{g}, \mathbf{g}^{\prime} \in \mathcal{G}^{n+1}$, we also have $\mathbb{P}_{n}^{w, \mathbf{a}}\left(t_{0}\right)=\mathbf{P}_{n}^{w, \mathbf{a}}\left(t_{0}\right)$.

By definitions (2.6) of $\widetilde{\mathbf{P}}_{n}^{w}(z)$ and (3.7) of $\mathbf{U}_{n}^{\mathbf{b}}(z)$, the $i j$-th block entry $\mathbf{Q}_{i j}^{w}(z)$ of the matrix $\mathbf{Q}_{n}^{w}(z):=\mathbf{U}_{n}^{\mathbf{b}}(z)^{*} \widetilde{\mathbf{P}}_{n}^{w}(z) \mathbf{U}_{n}^{\mathbf{b}}(z)$ equals

$$
\begin{aligned}
\mathbf{Q}_{i j}^{w}(z) & =\sum_{\ell=0}^{i} \sum_{k=0}^{j} \frac{1}{\ell ! k !} B_{i, i-\ell}(z)^{*}\left(\frac{\partial^{\ell+k}}{\partial \bar{z}^{\ell} \partial z^{k}} \frac{\mathrm{I}_{\mathcal{U}}-w(z)^{*} w(z)}{1-|z|^{2}}\right), B_{j, j-k}(z) \\
& =\frac{1}{i ! j !} \frac{\partial^{i+j}}{\partial \bar{z}^{i} \partial z^{j}}\left(B_{i}(z)^{*} \frac{\mathrm{I}_{\mathcal{U}}-w(z)^{*} w(z)}{1-|z|^{2}} B_{j}(z)\right),
\end{aligned}
$$

where the second equality follows from (6.1) by the Leibnitz rule. On the other hand, letting $f=\widetilde{L}_{z}^{(i)} g^{\prime}$ (for an arbitrary fixed $g^{\prime} \in \mathcal{G}$ ) in (6.6) gives

$$
\left\langle\widetilde{L}_{z}^{(j)} g^{\prime}, \widetilde{L}_{z}^{(i)} g\right\rangle_{H^{w}}=\frac{1}{i !}\left\langle\frac{\partial^{i}}{\partial \bar{z}^{i}}\left(\frac{B_{i}(z)^{*} \widetilde{L}_{z,-}^{(j)}(z)}{\bar{z}}\right) g^{\prime}, g\right\rangle_{\mathcal{G}}
$$


By (6.3) and (4.6), the bottom component of $\widetilde{L}_{z}^{(j)}(z)$ is equal to

$$
\widetilde{L}_{z,-}^{(j)}(z)=\frac{1}{j !} \frac{\partial^{j}}{\partial z^{j}}\left(\widetilde{K}_{z,-}(z) B_{j}(z)\right)=\frac{1}{j !} \frac{\partial^{j}}{\partial z^{j}}\left(\bar{z} \frac{1-w(z)^{*} w(\zeta)}{1-\bar{z} \zeta} B_{j}(z)\right) .
$$

Substituting the latter formula into the right hand side expression in (7.13) and comparing the result with (7.12), we conclude that

$$
\left\langle\widetilde{L}_{z}^{(j)} g^{\prime}, \widetilde{L}_{z}^{(i)} g\right\rangle_{H^{w}}=\left\langle\mathbf{Q}_{i j}^{w}(z) g^{\prime}, g\right\rangle_{\mathcal{G}} .
$$

Since the latter equalities hold for every $i, j=0, \ldots, n$ and every $g, g^{\prime} \in \mathcal{G}$, they can be equivalently written in terms of (7.2) as

$$
\left\langle\widetilde{\mathbf{L}}_{z} \mathbf{g}, \widetilde{\mathbf{L}}_{z} \mathbf{g}^{\prime}\right\rangle_{H^{w}}=\left\langle\mathbf{Q}_{n}^{w}(z) \mathbf{g}, \mathbf{g}^{\prime}\right\rangle, \quad \mathbf{g}, \mathbf{g}^{\prime} \in \mathcal{G}^{n+1} .
$$

By the second convergence in (7.4), we can pass in (7.9) to the limits as $z \widehat{\rightarrow} t_{0}$ to conclude that the weak limit $\mathbf{Q}_{n}^{w}\left(t_{0}\right)$ in (3.9) exists and satisfies

$$
\left\langle\mathbf{Q}_{n}^{w}\left(t_{0}\right) \mathbf{g}, \mathbf{g}^{\prime}\right\rangle=\left\langle\widetilde{\mathbf{L}}_{t_{0}} \mathbf{g}, \widetilde{\mathbf{L}}_{t_{0}} \mathbf{g}^{\prime}\right\rangle_{H^{w}} .
$$

Replacing $\mathrm{g}$ and $\mathrm{g}^{\prime}$ by $\mathrm{Mg}$ and $\mathrm{Mg}^{\prime}$ respectively, and making use of (7.11) and (7.6), we get

$$
\left\langle\mathbf{Q}_{n}^{w}\left(t_{0}\right) \mathbf{M g}^{\prime}, \mathbf{M g}\right\rangle=\left\langle\widetilde{\mathbf{L}}_{t_{0}} \mathbf{M g}, \widetilde{\mathbf{L}}_{t_{0}} \mathbf{M g}^{\prime}\right\rangle_{H^{w}}=\left\langle\mathbf{L}_{t_{0}} \mathbf{g}, \mathbf{L}_{t_{0}} \mathbf{g}^{\prime}\right\rangle_{H^{w}}=\left\langle\mathbf{P}_{n}^{w, \mathbf{a}}\left(t_{0}\right) \mathbf{g}, \mathbf{g}^{\prime}\right\rangle
$$

and conclude that $\mathbf{P}_{n}^{w, \mathbf{a}}\left(t_{0}\right)=\mathbf{M}^{*} \mathbf{Q}_{n}^{w}\left(t_{0}\right) \mathbf{M}$. Finally, the positivity of $\mathbf{P}_{n}^{w, \mathbf{a}}\left(t_{0}\right)$ follows from (7.6). This completes the proof of Theorem 3.1.

\section{Carathéodory-Julia condition and boundary behav- ior of functions in $H^{w}$}

In this section, we justify the terminology "boundary kernels" for $L_{t_{0}}^{(j)}$ and $\widetilde{L}_{t_{0}}^{(j)}$ by showing that these kernels reproduce (directional) nontangential boundary limits for the $j$-th derivative of functions $f \in H^{w}$.

Let us assume that condition (3.5) holds. Then the kernels $L_{t_{0}}^{(j)}$ and $\widetilde{L}_{t_{0}}^{(j)}$ are well-defined by formulas (5.9) and (6.10) for $j=0, \ldots, n$; furthermore, (7.1) holds. Then for every function $f=\left[\begin{array}{l}f_{+} \\ f_{-}\end{array}\right] \in H^{w}$ and every vector $g \in \mathcal{G}$, we can pass to the limits in (5.6) and (6.6) to get

$$
\begin{gathered}
\left\langle\lim _{z \rightarrow t_{0}}\left(\mathbf{a} f_{+}\right)_{j}(z), g\right\rangle_{\mathcal{G}}=\left\langle f, L_{t_{0}}^{(j)} g\right\rangle_{H^{w}}, \quad\left(\mathbf{a} f_{+}\right)_{j}(z):=\frac{\left(\mathbf{a} f_{+}\right)^{(j)}(z)}{j !}, \\
\left\langle\lim _{z \rightarrow t_{0}} \frac{1}{j !} \frac{d^{j}}{d \bar{z}^{j}}\left(\frac{B_{j}(z)^{*} f_{-}(z)}{\bar{z}}\right), g\right\rangle_{\mathcal{G}}=\left\langle f, \widetilde{L}_{t_{0}}^{(j)} g\right\rangle_{H^{w}} .
\end{gathered}
$$


Therefore, the weak limits

$$
\left(\mathbf{a} f_{+}\right)_{j}\left(t_{0}\right):=\mathrm{w}_{z} \lim _{z \rightarrow t_{0}}\left(\mathbf{a} f_{+}\right)_{j}(z), \quad C_{j}\left(t_{0}\right):=\mathrm{w}_{z} \lim _{z \rightarrow t_{0}} \frac{1}{j !} \frac{d^{j}}{d \bar{z}^{j}} \frac{B_{j}(z)^{*} f_{-}(z)}{\bar{z}}
$$

exist for $j=0, \ldots, n$. By (5.6), (8.1) and (7.1),

$$
\begin{aligned}
\left\|\left(\mathbf{a} f_{+}\right)_{j}(z)-\left(\mathbf{a} f_{+}\right)_{j}\left(t_{0}\right)\right\|_{\mathcal{Y}} & =\sup _{\|g\| \leq 1}\left|\left\langle\left(\mathbf{a} f_{+}\right)_{j}(z)-\left(\mathbf{a} f_{+}\right)_{j}\left(t_{0}\right), g\right\rangle_{\mathcal{G}}\right| \\
& =\left|\left\langle f, L_{z}^{(j)} g-L_{t_{0}}^{(j)} g\right\rangle_{H^{w}}\right| \\
& \leq\|f\|_{H^{w}} \cdot\left\|L_{z}^{(j)} g-L_{t_{0}}^{(j)} g\right\|_{H^{w}} \rightarrow 0
\end{aligned}
$$

as $z \widehat{\rightarrow} t_{0}$; and, therefore, the first limit in (8.3) exists in the strong sense. The strong convergence of the second limit in (8.3) follows in much the same way. Note also that, conversely, if the nontangential boundary limit

$$
\left(\mathbf{a} f_{+}\right)_{n}\left(t_{0}\right)=\frac{1}{n !}{ }_{z-\lim _{z \rightarrow t_{0}}}\left(\mathbf{a} f_{+}\right)^{(n)}(z) \quad \text { exists for every } f=\left[\begin{array}{l}
f_{+} \\
f_{-}
\end{array}\right] \in H^{w},
$$

then condition (3.5) holds. Indeed, by (5.6), (8.4) means that the limit $\lim _{z \rightarrow t_{0}}\left\langle f, L_{z}^{(n)} g\right\rangle_{H^{w}}$ exists for every $g \in \mathcal{G}$ and every $f \in H^{w}$. Fix $g$ and a nontangential neighborhood $\Gamma_{t_{0}, \alpha, \varepsilon}$ of $t_{0}$ (see (2.2)). Then $\left\langle f, L_{z}^{(n)} g\right\rangle_{H^{w}}$ is bounded for every $f \in H^{w}$ and $z \in \Gamma_{t_{0}, \alpha, \varepsilon}$, so by the uniform boundedness principle, $\left\|L_{z}^{(n)} g\right\|_{H^{w}}$ is uniformly bounded on $\Gamma_{t_{0}, \alpha, \varepsilon}$. By (5.8), we conclude that $\left\langle\mathbf{P}_{n, n}^{w, \mathbf{a}}(z) g, g\right\rangle_{\mathcal{G}}$ is uniformly bounded on $\Gamma_{t_{0}, \alpha, \varepsilon}$, which clearly implies (3.5). We have arrived at the following result.

Theorem 8.1. Let $w \in \mathcal{S}(\mathcal{U}, \mathcal{Y}), n \in \mathbb{Z}_{+}$and let a be an $\mathcal{L}(\mathcal{Y}, \mathcal{G})$-valued function analytic in a neighborhood of $t_{0} \in \mathbb{T}$. Then condition (3.5) is equivalent to (8.4). In this case, the strong limits

$$
\left(\mathbf{a} f_{+}\right)_{j}\left(t_{0}\right)=\mathrm{s}_{z} \lim _{z \rightarrow t_{0}}\left(\mathbf{a} f_{+}\right)_{j}(z) \quad \text { and } \quad C_{j}\left(t_{0}\right)=\mathrm{s}_{z \breve{\rightarrow} t_{0}} \frac{1}{j !} \frac{d^{j}}{d \bar{z}^{j}} \frac{B_{j}(z)^{*} f_{-}(z)}{\bar{z}}
$$

exist and are reproduced via the inner product of $H^{w}$ by formulas (8.1) and (8.2).

In the case where $\mathcal{G}=\mathcal{Y}$ and $\mathbf{a}(z) \equiv \mathrm{I}_{\mathcal{Y}}$, Theorem 8.1 simplifies as follows.

Theorem 8.2. Let $w \in \mathcal{S}(\mathcal{U}, \mathcal{Y}), n \in \mathbb{Z}_{+}$and $t_{0} \in \mathbb{T}$. Then (2.15) holds if and only if the nontangential boundary limit

$$
f_{+, n}\left(t_{0}\right)=\frac{1}{n !} \operatorname{w}_{z \rightarrow t_{0}} f_{+}^{(n)}(z) \quad \text { exists for every } f=\left[\begin{array}{l}
f_{+} \\
f_{-}
\end{array}\right] \in H^{w} .
$$


In this case, the strong limits

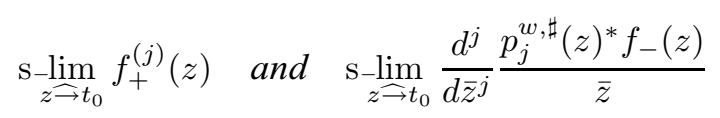

exist for $j=0, \ldots, n$, where $p_{j}^{w \sharp}$ are the polynomials defined in (2.12).

Making use of Theorem 8.2 and following the recipe of Remark 1.4, one can formulate the dual condition (2.20) in terms of boundary limits of $H^{w}$-functions. We omit the precise formulation and pass directly to the "combined" result.

Theorem 8.3. Let $w \in \mathcal{S}(\mathcal{U}, \mathcal{Y}), n \in \mathbb{Z}_{+}$and $t_{0} \in \mathbb{T}$. Then conditions (2.15) and (2.20) hold if and only if the nontangential boundary limit

$$
f_{n}\left(t_{0}\right):=\frac{1}{n !} \mathrm{w}_{z \rightarrow t_{0}} \lim _{z \rightarrow t_{0}}\left[\begin{array}{l}
\frac{d^{n}}{z^{n}} f_{+}(z) \\
\frac{d^{n}}{z^{n}} f_{-}(z)
\end{array}\right] \quad \text { exists for every } f=\left[\begin{array}{l}
f_{+} \\
f_{-}
\end{array}\right] \in H^{w} .
$$

In this case, the strong limits $f_{j}\left(t_{0}\right)$ exist for $j=0, \ldots, n$.

The latter results look somewhat more elegant in the context of the "onecomponent" de Branges-Rovnyak space $H(w)$, which was introduced in [7] as the space of all functions $h \in H_{2}^{+}(\mathcal{Y})$ such that

$$
\|h\|_{H(w)}^{2}:=\sup _{g \in H_{2}^{+}(\mathcal{U})}\left\{\|h+w g\|_{H_{2}^{+}(\mathcal{Y})}^{2}-\|g\|_{H_{2}^{+}(\mathcal{U})}^{2}\right\}<\infty .
$$

Alternatively, $H(w)$ can be defined as the reproducing kernel Hilbert space with reproducing kernel

$$
N(z, \zeta)=\frac{I_{\mathcal{Y}}-w(z) w(\zeta)^{*}}{1-z \bar{\zeta}}
$$

obtained via Aronszajn's construction [2]. The "two-component" space $H^{w}$ was originally introduced in [8] (in a slightly different, but equivalent, form) as the reproducing kernel Hilbert space with reproducing kernel

$$
R_{\zeta}(z)=\left[\begin{array}{cc}
N_{\zeta}(z) & \zeta \frac{w(z)-w(\zeta)}{z-\zeta \zeta *} \\
\bar{z} \frac{w(z)^{*}-w(\zeta)^{*}}{\bar{z}-\bar{\zeta}} & \bar{z} \zeta \frac{\mathrm{I}_{\mathcal{U}}-w(z)^{*} w(\zeta)}{1-\bar{z} \zeta}
\end{array}\right] .
$$

Its range space definition with the integral formula for the inner product presented in Section 4 comes from [12]. The space $H^{w}$ serves as the state space for the unitary realization of $w$; namely, there exist a unitary operator $\mathbf{U}=\left[\begin{array}{cc}A & B \\ C & D\end{array}\right]:\left[\begin{array}{c}H^{w} \\ \mathcal{U}\end{array}\right] \rightarrow\left[\begin{array}{c}H^{w} \\ \mathcal{Y}\end{array}\right]$ such that

$$
w(z)=D+z C\left(\mathrm{I}_{H^{w}}-z A\right)^{-1} B \quad \text { for every } z \in \mathbb{D} .
$$


Using equalities $\mathbf{U U}^{*}=\mathrm{I}_{H^{w} \oplus \mathcal{Y}}$ and $\mathbf{U}^{*} \mathbf{U}=\mathrm{I}_{H^{w} \oplus \mathcal{U}}$, one can factorize the kernel (8.9) in terms of the entries of $U$ as

$$
R_{\zeta}(z)=H(z) H(\zeta)^{*} \quad \text { where } H(z)=\left[\begin{array}{c}
C(\mathrm{I}-z A)^{-1} \\
\bar{z} B^{*}\left(\mathrm{I}-\bar{z} A^{*}\right)^{-1}
\end{array}\right]
$$

Theorem 8.4. Let $w \in \mathcal{S}(\mathcal{U}, \mathcal{Y}), n \in \mathbb{Z}_{+}$and let a be an $\mathcal{L}(\mathcal{Y}, \mathcal{G})$-valued function analytic at $t_{0} \in \mathbb{T}$. Then condition (3.5) holds if and only if the nontangential boundary limit

$$
\underset{z}{\mathrm{~W}-\lim }(\mathbf{a} h)^{(n)}(z) \quad \text { exists for every } h \in H(w) .
$$

In this case, the strong limits $\left.\mathrm{s}_{z \rightarrow t_{0}}(\mathbf{a} h)^{(j}\right)(z)$ exist for $j=0, \ldots, n$.

Proof. It follows from factorization (8.10) that for every $h \in H(w)$, there exists a function $\widetilde{h} \in H_{2}^{-}(\mathcal{U})$ such that $f=\left[\begin{array}{c}h \\ h\end{array}\right]$ belongs to $H^{w}$. Indeed, every $h \in H(w)$ is of the form $h(z)=C(\mathrm{I}-z A)^{-1} x$ for some $x \in H^{w}$, and we can simply take $\widetilde{h}(z)=\bar{z} B^{*}\left(\mathrm{I}-\bar{z} A^{*}\right)^{-1} x$. If we now assume that condition (3.5) holds, then (8.11) and the last statement in the theorem follow by Theorem 8.1.

To prove that (8.11) implies (3.5), we reproduce the arguments from the proof of Theorem 8.1, adapted to the single-component framework. Namely, we introduce

$$
T_{z}(t)=\frac{1}{n !} \frac{\partial^{n}}{\partial \bar{z}^{n}}\left(N(\zeta, z) \mathbf{a}(z)^{*}\right)
$$

(the analogue of $L_{z}^{(n)}($ see (5.1)) and note the equalities

$$
\frac{1}{n !}\left\langle(\mathbf{a}(z) h(z))^{(n)}, g\right\rangle_{\mathcal{G}}=\left\langle h, T_{z} g\right\rangle_{H(w)}
$$

and

$$
\left\|T_{z} g\right\|_{H(w)}^{2}=\left\langle\mathbf{P}_{n n}^{w, \mathbf{a}}(z) g, g\right\rangle_{\mathcal{G}}
$$

holding for every $g \in \mathcal{G}$ and $h \in H(w)$. To prove (8.12) and (8.13), we repeat the proofs of Lemma 5.1 and Lemma 5.2, respectively, and use the fact that $N(z, \zeta)$ is a reproducing kernel for $H(w)$. Assuming that condition (8.11) holds, we conclude by (8.12) that the limit $\lim _{z \widehat{\rightarrow} t_{0}}\left\langle h, T_{z} g\right\rangle_{H(w)}$ exists for every $g \in \mathcal{G}$ and every $h \in H(w)$. Then $\left\langle h, T_{z} g\right\rangle_{H(w)}$ is bounded for every fixed $h \in H(w)$ and every $z$ from a fixed nontangential neighborhood $\Gamma_{t_{0}, \alpha, \varepsilon}$ of $t_{0}$. By the uniform boundedness principle, $\left\|T_{z} g\right\|_{H(w)}$ is uniformly bounded on $\mathcal{U}$, which implies (3.5) by (8.13). 
Recently, Fricain and Mashreghi [10] showed that if $\mathbf{a}(z) \equiv 1$ and

$$
w(z)=\prod_{k} \frac{\bar{a}_{k}}{a_{k}} \cdot \frac{z-a_{k}}{1-z \bar{a}_{k}} \cdot \exp \left\{-\int_{0}^{2 \pi} \frac{e^{i \theta}+z}{e^{i \theta}-z} d \mu(\theta)\right\}
$$

is a scalar Schur-class function, then (8.11) holds if and only if

$$
\sum_{k} \frac{1-\left|a_{k}\right|^{2}}{\left|t_{0}-a_{k}\right|^{2 n+2}}+\int_{0}^{2 \pi} \frac{d \mu(\theta)}{\left|t_{0}-e^{i \theta}\right|^{2 n+2}}<\infty
$$

(in case $w$ is inner, this result was established in the remarkable paper [1]). Thus, in the scalar valued case, condition (8.14) appears to be equivalent to the Carathéodory-Julia condition (1.9). A natural question concerning the operator valued analogue of (8.14) (supposedly equivalent to condition (3.5)) will be presented on a separate occasion.

\section{REFERENCES}

[1] P. R. Ahern and D. N. Clark, Radial limits and invariant subspaces, Amer. J. Math. 92 (1970), 332-342.

[2] N. Aronszajn, Theory of reproducing kernels, Trans. Amer. Math. Soc. 68 (1950), 337-404.

[3] V. Bolotnikov and H. Dym, On boundary interpolation for matrix valued Schur functions, Mem. Amer. Math. Soc. 181 (2006), no. 856.

[4] V. Bolotnikov and A. Kheifets, A higher order analogue of the Carathéodory-Julia theorem, J. Funct. Anal. 237 (2006), 350-371.

[5] V. Bolotnikov and A. Kheifets, Carathéodory-Julia type conditions and symmetries of boundary asymptotics for analytic functions on the unit disk, Math. Nachr., to appear.

[6] V. Bolotnikov and A. Kheifets, The higher order Carathéodory-Julia theorem and related boundary interpolation problems, in: Recent Advances in Matrix and Operator Theory, Birkhäuser, Basel, 2007.

[7] L. de Branges and J. Rovnyak, Square Summable Power Series, Holt, Rinehart and Winston, New-York, 1966.

[8] L. de Branges and L. Shulman, Perturbations of unitary transformations, J. Math. Anal. Appl. 23 (1968), 294-326.

[9] H. Dym, J Contractive Matrix Functions, Reproducing Kernel Spaces and Interpolation, Amer. Math. Soc., Providence, RI, 1989.

[10] E. Fricain and J. Mashreghi, Boundary behavior of functions in the de Branges-Rovnyak spaces, Complex Anal. Oper. Theory 2 (2008), 87-97.

[11] I. V. Kovalishina, Carathéodory-Julia theorem for matrix-functions, Teoriya Funktsii, Funktsianal'nyi Analiz i Ikh Prilozheniya 43 (1985), 70-82. English translation in: Journal of Soviet Mathematics 48(2) (1990), 176-186.

[12] N. K. Nikolskiĭ and V. I. Vasyunin, A unified approach to function models, and the transcription problem, in: The Gohberg Anniversary Collection, Vol. II, 405-434, Birkhäuser, Basel, 1989. 
[13] D. Sarason, Angular derivatives via Hilbert space, Complex Variables Theory Appl. 10 (1988), $1-10$.

[14] D. Sarason, Sub-Hardy Hilbert Spaces in the Unit Disk, Wiley, New York, 1994.

\author{
Vladimir Bolotnikov \\ DEPARTMENT OF MATHEMATICS, \\ THE COLLEGE OF WILliaM AND MARY, \\ WILLIAMSBURG, VA 23187-8795, USA \\ email: vladi@math.wm.edu \\ Alexander Kheifets \\ DEPARTMENT OF MATHEMATICS, \\ UNIVERSITY OF MASSACHUSETTS LOWELL, \\ LOWELL, MA, 01854, USA \\ email: Alexander_Kheifets@uml.edu
}

(Received July 30, 2007 and in revised form December 17, 2007) 